\title{
Linking epigenetic dysregulation, mitochondrial impairment, and metabolic dysfunction in SBMA motor neurons
}

\author{
Naemeh Pourshafie, ${ }^{1,2}$ Ester Masati, ${ }^{1}$ Eric Bunker, ${ }^{1}$ Alec R. Nickolls, ${ }^{1,3,4}$ Parisorn Thepmankorn, ${ }^{1}$ \\ Kory Johnson, ${ }^{1}$ Xia Feng, ${ }^{1,5}$ Tyler Ekins, ${ }^{3,6}$ Christopher Grunseich, ${ }^{1}$ and Kenneth H. Fischbeck ${ }^{1}$ \\ ${ }^{1}$ Neurogenetics Branch, National Institute of Neurological Disorders and Stroke, NIH, Bethesda, Maryland, USA. \\ ${ }^{2}$ George Washington University, Institute of Biomedical Sciences, Washington, DC, USA. ${ }^{3}$ Brown University, Department of \\ Neuroscience, Providence, Rhode Island, USA. ${ }^{4}$ National Center for Complementary and Integrative Health, NIH, Bethesda, \\ Maryland, USA. 5Johns Hopkins University, Department of Psychiatry and Behavioral Sciences, Baltimore, Maryland, \\ USA. ${ }^{6}$ Program in Developmental Neuroscience, Eunice Kennedy-Shriver National Institute of Child Health and Human \\ Development, NIH, Bethesda, Maryland, USA.
}

Spinal and bulbar muscular atrophy (SBMA) is a neuromuscular disorder caused by a polyglutamine expansion in the androgen receptor (AR). Using gene expression analysis and ChIP sequencing, we mapped transcriptional changes in genetically engineered patient stem cell-derived motor neurons. We found that transcriptional dysregulation in SBMA can occur through AR-mediated histone modification. We detected reduced histone acetylation, along with decreased expression of genes encoding compensatory metabolic proteins and reduced substrate availability for mitochondrial function. Furthermore, we found that pyruvate supplementation corrected this deficiency and improved mitochondrial function and SBMA motor neuron viability. We propose that epigenetic dysregulation of metabolic genes contributes to reduced mitochondrial ATP production. Our results show a molecular link between altered epigenetic regulation and mitochondrial metabolism that contributes to neurodegeneration.

Conflict of interest: The authors have declared that no conflict of interest exists.

Copyright: (c) 2020, American Society for Clinical Investigation.

Submitted: January 16, 2020

Accepted: May 21, 2020

Published: July 9, 2020.

Reference information: /CI Insight. 2020;5(13):e136539.

https://doi.org/10.1172/jci.

insight.136539.

\section{Introduction}

Spinal and bulbar muscular atrophy (SBMA) is an X-linked neuromuscular disorder characterized by lower motor neuron and skeletal muscle degeneration (1). The signs of lower motor neuron disease include weakness, atrophy, and fasciculations in the bulbar and extremity muscles of SBMA patients (2). SBMA is caused by the expansion of a polyglutamine-encoding CAG repeat in the first exon of the androgen receptor (AR) gene (3). The AR is a ligand-dependent transcription factor, which binds to specific DNA sequences and regulates transcription (4). It has been well established that transcriptional dysregulation in SBMA involves both a partial loss of the AR's transactivation function (5) and a toxic gain of function of the mutant AR (6). Previous studies have shown disruption of multiple cellular processes, including transcriptional dysregulation and impairment in mitochondrial function, as contributing to neuronal dysfunction in SBMA and other polyglutamine diseases $(7,8)$.

Mitochondria integrate metabolic pathways to generate ATP by oxidative phosphorylation. These dynamic organelles undergo structural and functional modification to adapt to environmental changes, such as nutrient availability and cellular stress (9). Previous work has shown decreased mitochondrial membrane potential and increased redox imbalance in cell models of SBMA (10). Further evidence suggests that the mutant AR causes alterations in energy metabolism and gene expression pathways involved in regulating metabolic homeostasis. Dysregulation of genes affecting skeletal muscle metabolism in SBMA (11, 12) - along with the clinical findings of excess triacylglycerol accumulation in the liver, elevated glucose and insulin levels, and low levels of serum insulin-like growth factor (IGF-1) (13) - all indicate alteration in metabolic processes in multiple organ systems where AR is expressed.

Here, we study a potentially new mode of AR-dependent transcriptional dysregulation in SBMA and its link to mitochondrial impairment. To accomplish this, we used an RNA sequencing (RNA-seq) analysis integrated with ChIP of AR protein and histone markers using SBMA, control, and AR-KO human 
induced pluripotent stem cell-derived (iPSC-derived) motor neuron-like cells. Histone 3 lysine 27 acetylation (H3K27ac) ChIP showed alterations in histone acetylation influencing the gene expression landscape in the SBMA cells.

We found that reduced expression of compensatory metabolic genes in SBMA is associated with a corresponding reduction of histone acetylation. Biochemical studies of the SBMA motor neuron-like cells showed reduced AMPK signaling and reduced intracellular levels of acetyl-CoA, a key intermediate metabolite essential to mitochondrial function. We found that increasing intracellular acetyl-CoA by pyruvate supplementation improves SBMA mitochondrial function and SBMA motor neuron survival. Our results indicate that alteration in histone modifications at genes involved in compensatory metabolic pathways exacerbates mitochondrial dysfunction in SBMA.

\section{Results}

SBMA iPSC-derived motor neurons show cellular toxicity and neurodegeneration. We previously generated and characterized iPSCs from fibroblasts of SBMA and unaffected control individuals (14). We stably transfected these iPSCs with a doxycycline-inducible cassette containing human NGN2/ISL1/LHX3 (hNIL) genes with TALEN or CRISPR/Cas9-mediated integration (15). We used CRISPR/Cas9 to knock out AR in both an SBMA and control iPSC lines to distinguish effects of AR toxic gain-of-function and lossof-normal function effects in the cells. In total, we transfected 4 SBMA, 3 control, and 2 AR-KO iPSC lines with the hNIL cassette (Supplemental Figure 1A; supplemental material available online with this article; https://doi.org/10.1172/jci.insight.136539DS1). Four days after doxycycline induction (dpi), the iPSC-derived motor neurons (iMNs) expressed the general neuronal marker $\beta$ III tubulin (TUJ1), and 2 transcription factors normally expressed in motor neurons HB9 (MNX1) and ISL1 (Figure 1A). All cell lines showed similar differentiation efficiency, with over $90 \%$ cells costained with HB9, ISL1, and TUJ1 within 6 days in culture (Figure 1, B and C). The differentiation of these iMNs resulted in cells with expression of motor neuron genes and downregulation of the pluripotency-associated factors SOX1 and Nanog (Supplemental Figure 1B). Quantitative PCR (qPCR) analysis of 15 genes selected to represent spinal motor neuron maturation and development (16) showed differentiation consistent with a spinal motor neuron cell type (Supplemental Figure 1C). The electrophysiological properties of control iMNs 28 dpi were determined by whole-cell patch clamp recording. iMNs fired action potentials with the injection of depolarizing currents. Action potentials were ablated with tetrodotoxin (TTX), indicating that they are mediated by TTX-sensitive voltage-gated sodium channels (Supplemental Figure 1D).

We next evaluated these iMNs for AR expression and nuclear translocation of the AR in response to dihydrotestosterone (DHT). Consistent with previous findings (14), AR mRNA expression was not different in SBMA and control iMNs (Supplemental Figure 1E). Nuclear fractionation followed by Western blot (Supplemental Figure 1F) and immunofluorescent staining (Supplemental Figure 1G) showed that both WT and mutant AR localized into the nucleus with ligand binding.

An important manifestation of SBMA is motor neuron degeneration. To determine whether the SBMA iMNs recapitulate this feature, we assessed cell morphology and neuronal cell death in 3 patient, 3 control, and 2 AR-KO lines over time after DHT treatment. The SBMA cells had degenerative features, with more cell debris detected in the culture media compared with control and AR-KO cells (Supplemental Figure 2A). In addition, more SBMA iMNs were $\mathrm{TUNEL}^{+}$(Supplemental Figure 2B) and had significantly higher levels of activated caspase-3 than control iMNs (Supplemental Figure 2, C-E), indicating apoptosis in the disease cells. These results show that cellular stress and death are substantially mediated by a toxic gain of function of the mutant AR.

Depletion of intracellular ATP correlates with progressive degeneration in SBMA iMNs. To determine whether the SBMA iMNs recapitulate this disease phenotype, we measured ATP production by measurement of extracellular acidification and oxygen consumption using the bioenergetic extracellular flux assay (Agilent). We performed this functional assay on immature ( $2 \mathrm{dpi}$ ) and differentiated motor neurons ( $4 \mathrm{dpi})$ to determine whether changes in ATP production rate correlate with disease progression. Furthermore, we measured the rates of glycolysis and mitochondrial ATP production separately to investigate the relative contribution of these metabolic pathways to energy production in the cells. Energy production declined in SBMA as the cells matured. The SBMA iMNs had a significantly lower mitochondrial ATP production rate compared with control cells at $4 \mathrm{dpi}(P<0.0001)$. The rate of mitochondrial ATP production was less affected in the AR-KO cells and did not change significantly with time (Figure 1D). In accordance with 
A
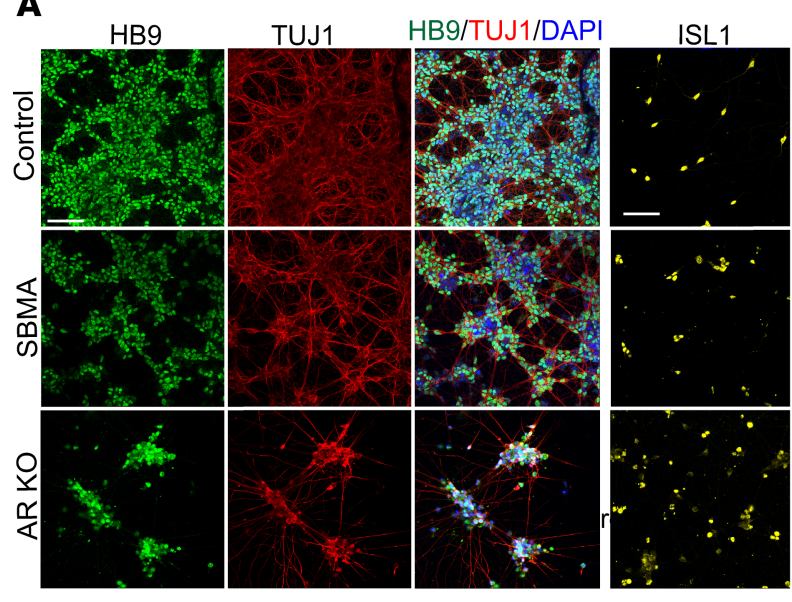

TUJ1 ISL1/TUJ1/DAPI
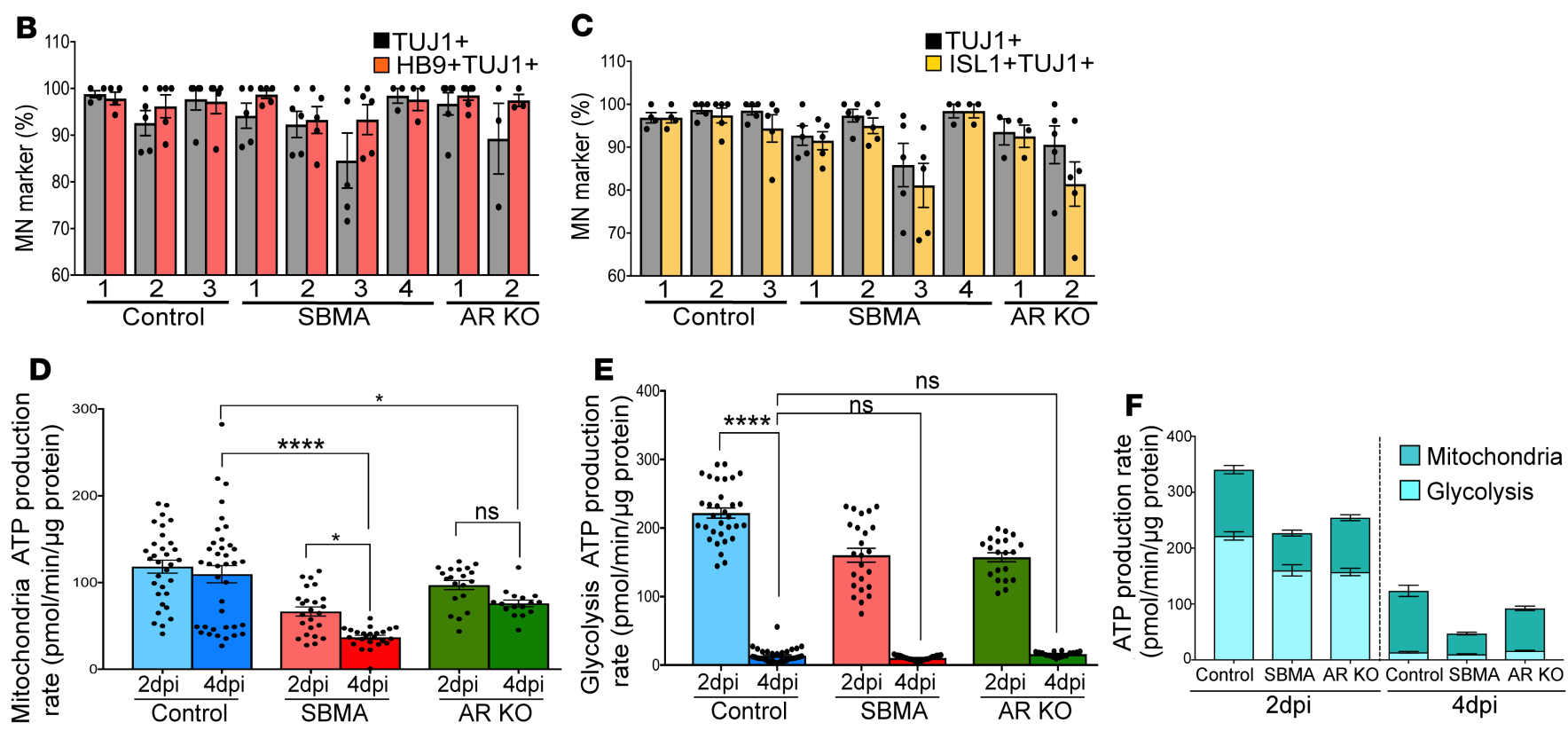

G

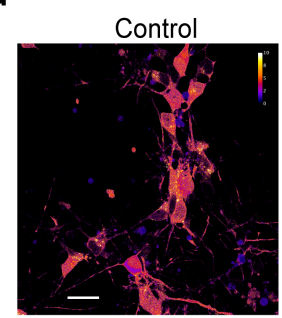

SBMA

AR KO
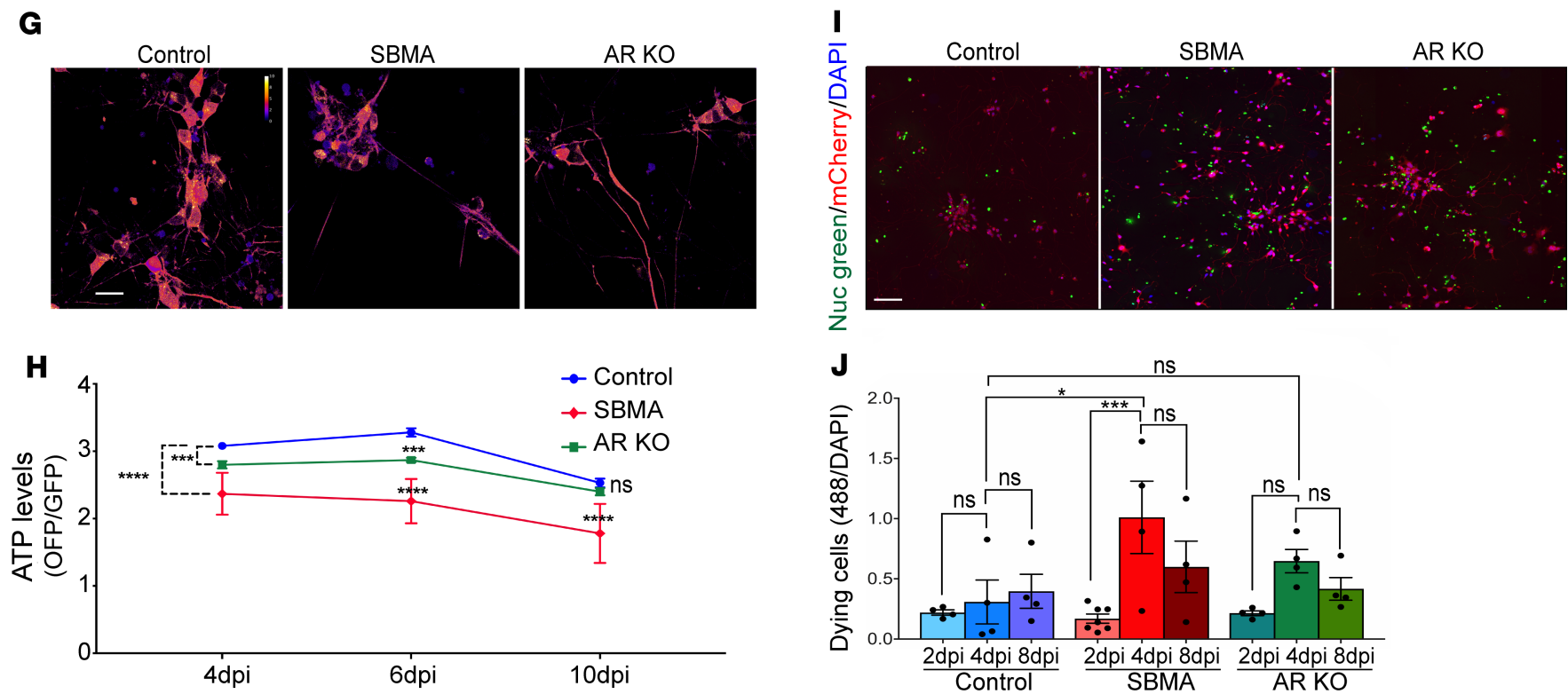
Figure 1. iMNs differentiated from SBMA iPSCs show increased cellular stress and cell death. (A) Representative images of iMNs (6 dpi) expressing HB9, ISL1, and TUJ1. Scale bars: $75 \mu \mathrm{m}$. (B and C) Percentage of HB9+/TUJ1+ (B) and ISL1+/TUJ1+ (C), assessed by immunostaining. $n=4-5$ per cell line. (D-F) Bioenergetic extracellular flux analysis (Seahorse assay) on iMNs normalized to total cellular protein concentration. (D) Rate of ATP production during oxidative phosphorylation (MitoATP production rate). (E) Rate of ATP production in the glycolytic pathway (glycoATP production rate). (F) The sum of the glycolytic and mitochondrial ATP production rates (total ATP production rate). $n=16-38$ wells/group. (G) Ratiometric pseudocolor images of GoATEAM expressed in iMNs. ATP sensors were introduced into iMNs using lentivirus 4 days before taking the first image. (H) Comparison of orange/green fluorescence emission ratio of GoATEAM at different time points. The ratio was calculated from fluorescence images. Plates were seeded at the same density, and live images were taken from the same plate over time. On average, 30 images per cell line were used for calculating the ratio at each time point. (I) Representative images of dying cells with less plasma membrane integrity were detected with a florescent stain in real time. NucGreen dead 488 (green), iMNs expressing the hNIL-mCherry plasmid (red), and DAPI (blue). (J) Comparison of GFP/DAPI emission ratio of NucGreen dead at different time points. The ratio was calculated from fluorescence images. $n=4-6$ per group. All experiments were performed on $N=3 \mathrm{SBMA}, N=3$ control, and $N=3$ AR-KO. Error bars show mean $\pm \mathrm{SE} ;{ }^{*} P<0.05$, ${ }^{* *} P<0.001$, ${ }^{* * *} P<0.0001$. One-way ANOVA followed by Bonferroni's multiple comparisons test. iMNs were treated with $10 \mathrm{nM}$ DHT. Scale bars: $25 \mu \mathrm{m}$ (C) and $40 \mu \mathrm{m}(\mathbf{I})$.

previous findings (17), the motor neuron-like cells switched from glycolytic to oxidative metabolism with differentiation (Figure 1E). These results show that motor neurons rely on mitochondria for energy synthesis, and in the case of mitochondrial energy deficiency, the SBMA cells are unable to use glycolysis to fully compensate for ATP loss (Figure 1F).

To ascertain whether mitochondrial impairment in SBMA cells leads to an ATP shortage, we used a fluorescence resonance energy transfer-based ATP indicator (GoATeam) to measure the relative levels of ATP in the motor neurons in real-time (18). This ATP indicator has a high specificity for ATP over other nucleotides and changes the fluorescence emission, with a higher orange-to-green fluorescence emission ratio $(560 / 510 \mathrm{~nm})$ reflecting higher ATP levels. Consistent with the Seahorse assay results, the relative amount of ATP decreased significantly in SBMA cells over time $(P<0.0001$; Figure $1, \mathrm{G}$ and H). Interestingly, the AR-KO iMNs showed low levels of ATP at 4 dpi compared with control, but the ATP levels in these cells did not markedly decline with time. This suggests that, despite compromised mitochondrial activity, the AR-KO iMNs can regulate bioenergetic homeostasis to keep ATP levels constant, whereas the SBMA iMNs have impaired homeostasis. By $10 \mathrm{dpi}$, the AR-KO cells showed the same relative levels of ATP as controls, while the SBMA iMNs were significantly depleted $(P<0.0001$; Figure $1 \mathrm{H})$. Next, we examined whether there is a time-dependent correlation between motor neuron degeneration and energy depletion. We assessed real-time survival of SBMA iMNs over time, using a DNA-binding dye (NucGreen 488) that stains degenerating cells by penetrating the compromised cell membranes. More SBMA iMNs were permeable to this dye compared with AR-KO and the controls, and the number of permeable SBMA cells increased from 2-4 dpi $(P<0.01$; Figures 1 , I and J). Loss of membrane integrity and ATP production followed a similar time course in the SBMA cells, and the changes in these cells were more marked than in the AR-KO cells, consistent with a toxic gain of function by mutant AR in SBMA.

SBMA and control motor neurons show distinct AR chromatin binding profiles. AR transcriptional regulation is tightly controlled by epigenetic regulation (19). To investigate potential differences in the epigenetic landscape between WT AR and mutant AR, we used ChIP-seq for AR and active histone markers. Using AR ChIP-seq across 3 cell lines each from SBMA and control iMNs and 1 AR-KO, we mapped overlap in the genomic landscape of AR binding with the patterns of H3K4me1, associated with regulatory regions and enhancers; $\mathrm{H} 3 \mathrm{~K} 4 \mathrm{me} 3$, associated with promoters; and $\mathrm{H} 3 \mathrm{~K} 27 \mathrm{ac}$, associated with active chromatin modification $(20,21)$. We studied SBMA iMNs with 51-55 CAG repeats to minimize variability among the disease cell lines. We found a total of 5,852 AR peaks after normalization to AR-KO, which served as a negative control (Supplemental Figure 3A), with some differential binding between the WT and mutant AR (Supplemental Figure 3B). We selected peaks within $\pm 10 \mathrm{~kb}$ from the refseq transcriptional start sites (TSS) to incorporate proximal events. We assessed the genomewide distribution of AR at loci coprecipitated by H3K4me3, H3K4me1, and H3K27ac. Interestingly, H3K27ac enrichment around WT AR-occupied regions was characterized by a unimodal distribution, whereas a bimodal distribution profile was found around polyQ AR-occupied regions (Figure 2A). Furthermore, the pattern of AR colocalization with H3K4me3-associated promoter and H3K4me1-associated enhancer regions was highly distinct (Supplemental Figure 3C). Collectively, these data suggest differential genomic binding between the WT and polyQ AR.

Repression of metabolic-related genes in SBMA is associated with reduced histone acetylation at their regulatory regions. WT AR colocalization with $\mathrm{H} 3 \mathrm{~K} 27 \mathrm{ac}$ peaks (Figure $2 \mathrm{~A}$ ) implies a correlation between the AR and 
A
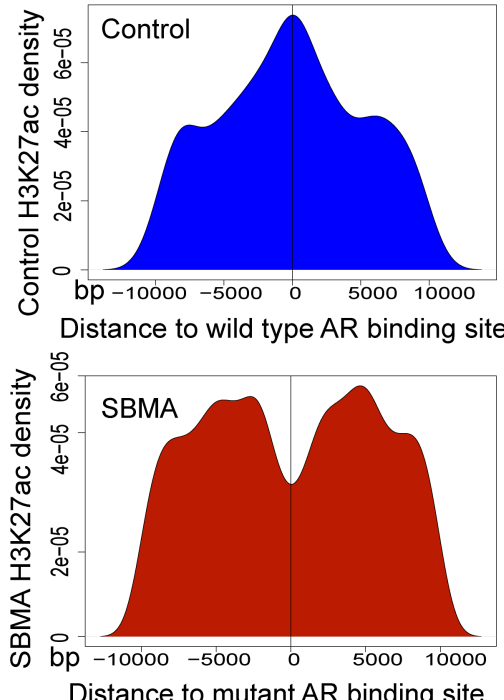

Distance to mutant $A R$ binding site
$\mathbf{B}$

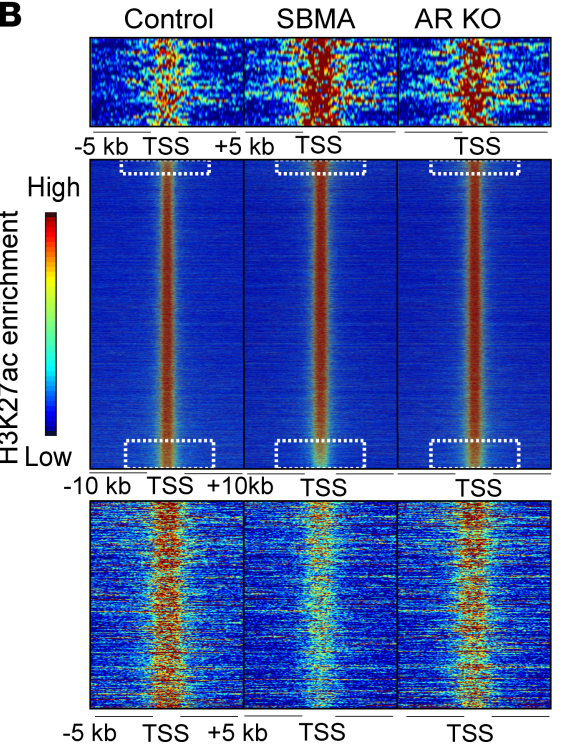

C AR KO/Control SBMA/Control

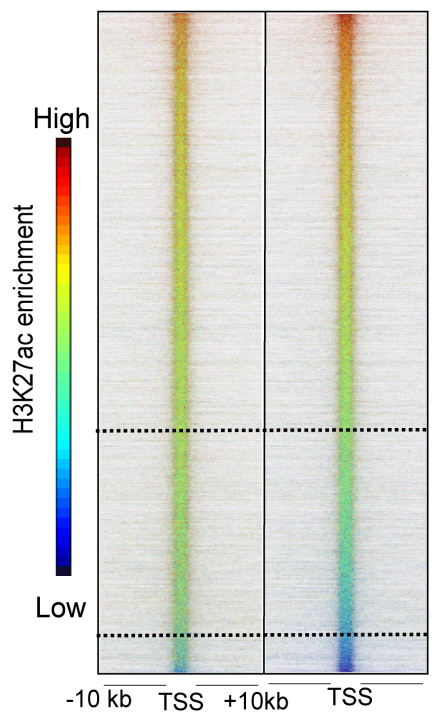

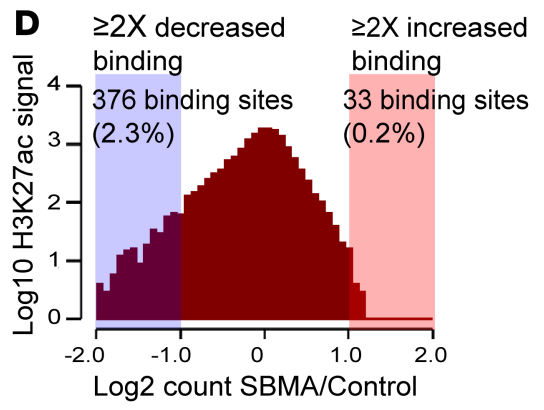

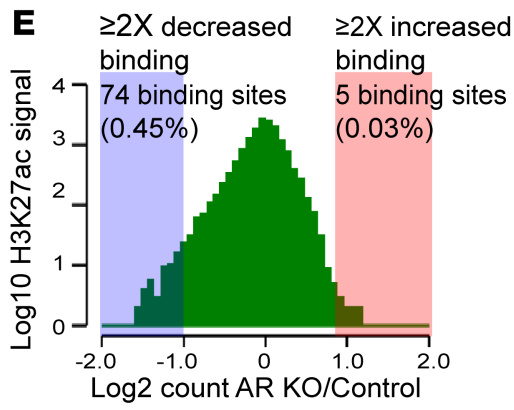

Figure 2. Reduction in H3K27ac binding corresponds to repression in metabolic genes. (A) H3K27ac peak distribution near WT AR (top) and mutant AR (bottom) peaks. The H3K27ac signal is shown over a scaled window of $\pm 10 \mathrm{~kb}$ from the AR peak. (B) Heatmap of H3K27ac occupancy signals at \pm 10 $\mathrm{kb}$ region centered around all Refseq TSS ranked by increasing H3K27ac signal intensity in SBMA over control. The height of the heatmap shows 16,118 H3K27ac binding regions across the genome. (C) Ratiometric heatmap comparing H3K27ac signals. Signals are ranked by increased H3K27ac signal intensity in SBMA/control. Demarcated regions represent H3K27ac occupancy signals with greater than 1.5-fold and 2-fold reduced binding. (D and E) Histograms representing 16,118 H3K27ac signals between SBMA/control and ARKO/control. Boxed regions represent H3K27ac binding regions with greater than 2-fold enrichment (red) and greater than 2-fold reduced binding (blue). iMNs were treated with $10 \mathrm{nM}$ DHT.

H3K27ac binding events. H3K27ac marks both active enhancers and promoters and mediates epigenetic regulation of gene expression. Consequently, reduced histone acetylation has been implicated in the pathophysiology of various neurological disorders by eliciting pathological gene expression programs (22, 23). Evidence has shown that CREB binding protein-mediated (CBP-mediated) transcription is impaired in SBMA and that histone deacetylase inhibitors reduce SBMA cell death (6). We profiled H3K27ac by ChIP-seq and identified a total of $16,118 \mathrm{H} 3 \mathrm{~K} 27 \mathrm{ac}$ binding regions across the genome. We assessed the genome-wide distribution of $\mathrm{H} 3 \mathrm{~K} 27 \mathrm{ac}$ at loci cooccupied by $\mathrm{H} 3 \mathrm{~K} 4 \mathrm{me} 3$ and $\mathrm{H} 3 \mathrm{~K} 4 \mathrm{me}$. In agreement with previously reported genomic studies $(20,21)$, H3K27ac peaks colocalized with H3K4me1- and H3K4me3-occupied regions in both SBMA and control iMNs (Supplemental Figure 4, A and B). We analyzed the enrichment of H3K27ac signals within $\pm 10 \mathrm{~kb}$ of TSS to identify differentially enriched H3K27ac binding sites (Figure 2, B and C). The H3K27ac binding sites $(2.3 \%$ and $0.2 \%$ ) showed more than 2 -fold reduced or increased binding in SBMA iMNs compared with control, respectively (Figure 2D). Notably, only a small fraction of these regions displayed a more than 2-fold differential enrichment in AR-KO compared with control (Figure 2E). These data show that the AR-KO has a similar pattern of H3K27ac enrichment compared with control iMNs.

Reduced H3K27ac binding in SBMA could lead to a reduction of gene expression. In support of this, $\mathrm{H} 3 \mathrm{~K} 27 \mathrm{ac}$ and RNA polymerase II generally cooccupied the same genomic loci (Supplemental Figure 4C). To examine whether the reduced acetylation of $\mathrm{H} 3 \mathrm{~K} 27 \mathrm{ac}$ is accompanied by reduced gene expression, we performed RNA-seq on 4 SBMA, 4 control, and 3 AR-KO iMNs (Supplemental Figure 1A). 
A $\quad$ GO for $2 x$ reduced $\mathrm{H} 3 \mathrm{~K} 27$ ac binding

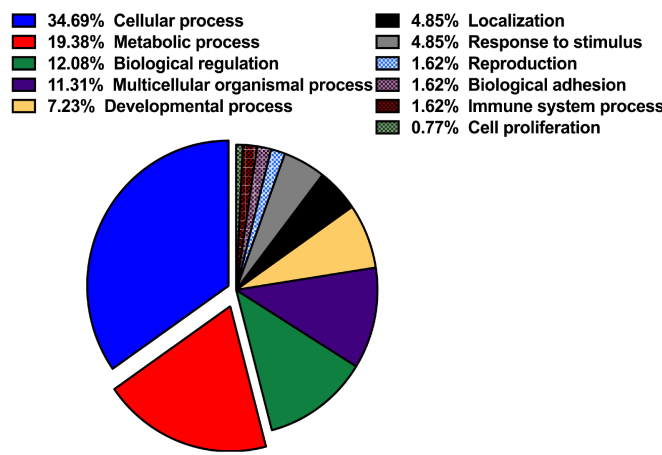

C

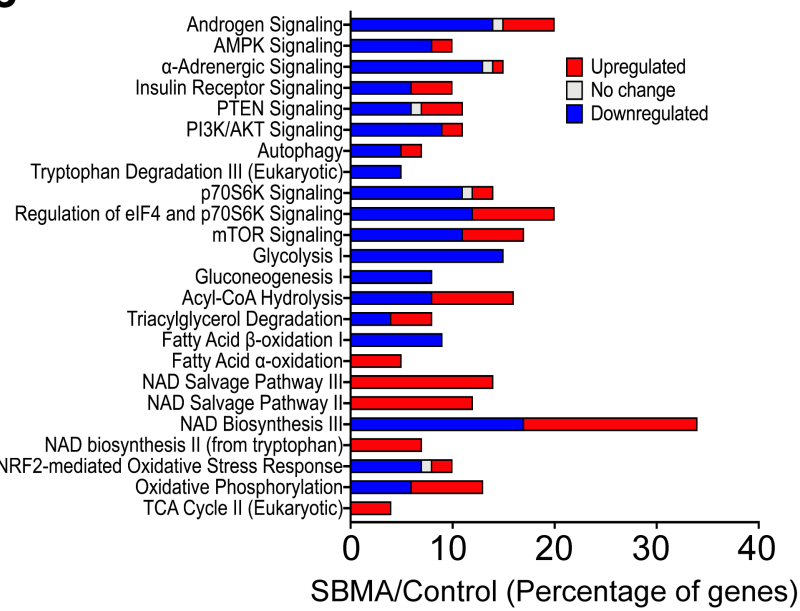

B
IPA for 2x reduced H3K27ac binding of Metabolic genes
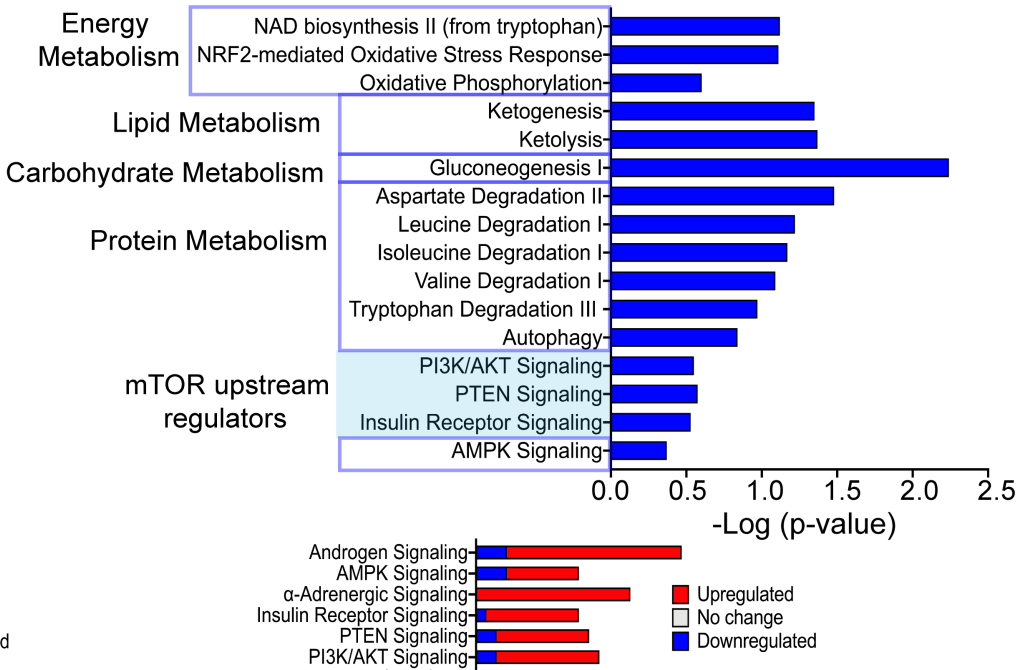

Tryptophan Degradation III (Eukaryotic)- I

p70S6K Signaling-
Regulation of elF4 and p70S6K Signaling-

mTOR Signaling

Gluconeogenesis

Acyl-CoA Hydrolys - 1

Triacylglycerol Degradation-

Fatty Acid $\beta$-oxidation I-

Fatty Acid a-oxidation-

NAD Salvage Pathway III-

NAD Salvage Pathway II-

NAD Biosynthesis III-

NRF2

Oxidative Phosphorylation-

TCA Cycle II (Eukaryotic) -

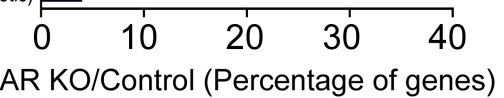

Figure 3. Alteration in metabolic pathways mediated by reduced H3K27ac. (A) Genome ontology (GO) enrichment analysis of the closest gene with greater than 2-fold reduced H3K27ac using Panther. (B) Ingenuity Pathway Analysis (IPA) of the closest genes with greater than 2-fold reduced H3K27ac binding. Analysis demonstrating key metabolic pathways identified in the GO enrichment analysis in. mTOR upstream regulatory pathways are highlighted in blue. (C) IPA of RNA-seq data showing percentage of genes upregulated (red), genes downregulated (blue), or genes with no change (gray) within each metabolic pathway. iMNs were treated with $10 \mathrm{nM}$ DHT.

We selected 4-6 dpi iMNs for this screen because, at this time point, SBMA iMNs had begun to show signs of degeneration (Supplemental Figure 2A).

Reduced expression of genes corresponded with decreased H3K27ac enrichment (Supplemental Figure 5A and Supplemental Table 1). Gene ontology (GO) analysis of biological processes for genes with 2-fold reduced H3K27ac binding near their TSS showed that over 19\% of these genes are involved in metabolic processes (Figure 3A). Canonical pathway analysis of the annotated genes related to metabolic processes revealed that many associated with key regulators of cellular energy homeostasis pathways (AMPK and mTORC upstream regulators), and catabolic pathways such as ketogenesis, gluconeogenesis, and degradation of amino acids (Figure 3B), providing a link between changes in intracellular metabolism and altered epigenetic gene regulation in SBMA. These data are in accordance with previous findings that histone acetylation levels are responsive to nutrient availability (24). Pathway analysis of upregulated genes with more than 2-fold increased $\mathrm{H} 3 \mathrm{~K} 27 \mathrm{ac}$ also showed enrichment of genes associated with the protein ubiquitination pathway, cAMP-mediated signaling, and GPCRs (Supplemental Figure 4D).

Alteration of metabolic pathways in SBMA iMNs. The similar distribution pattern of H3K27ac between the control and AR-KO iMNs indicates that reduced histone acetylation is likely mediated by AR toxic gain of function rather than by loss of normal function (Figure 2, B and C). Notably, differential H3K27ac enrichment occurred near the TSS of genes associated with energy homeostasis and metabolism (Figure 3B). To extend these findings, we examined the RNA-seq data to assess the consequence of the reduced acetylation. Ingenuity pathway analysis (IPA; QIAGEN) of genes with significantly different expression in the SBMA and AR-KO iMNs showed altered activity of genes that influence energy homeostasis (Supplemental Figure 5B). 
Among the top pathways were genes associated with mTOR signaling and direct downstream targets, eukaryotic translation initiation factor 4E (eIF4) and ribosomal protein S6 kinases (P70S6K), which control protein synthesis (25). Protein synthesis requires utilization of energy (26). In response to changes in intracellular ATP levels, cells regulate protein synthesis through the mTOR signaling pathway (Supplemental Figure 5C) (17). Therefore, alteration of mTOR signaling in SBMA iMNs suggests disruption in cellular ATP levels and altered mitochondrial oxidative phosphorylation and ATP synthesis. In-depth analysis of the RNA-seq data for metabolic genes and pathways showed that a higher percentage of genes associated with these metabolic processes (e.g., AMPK signaling and gluconeogenesis) are downregulated in SBMA and upregulated in AR-KO iMNs (Figure 3C). This suggests that these cells are unable to effectively regulate adaptive responses to bioenergetic collapse. Collectively, these data suggest that the AR-KO iMNs can regulate and maintain key metabolic and cellular processes to sustain their bioenergetic needs, whereas bioenergetic adaptation is compromised in the presence of the mutant AR in SBMA iMNs.

Decreased AMPK signaling in response to low ATP levels prevents sufficient metabolic adaptation in SBMA. To investigate potential metabolic changes, we used an ELISA-based antibody array to evaluate the phosphorylation of upstream and downstream proteins of AMPK. AMPK is a main intracellular energy sensor and regulates cell metabolism in response to changes in ATP levels. Once phosphorylated, activated AMPK functions broadly to inhibit anabolic pathways and stimulates catabolic pathways (27). Notably, AMPK hyperphosphorylation has been reported in the neurons of patients with Alzheimer and Huntington diseases $(28,29)$.

AMPK phosphorylation was upregulated on Thr172 in both SBMA and AR-KO iMNs compared with control (Figure 4A), which is a response to low intracellular ATP levels (30). Thr172 phosphorylation is required for AMPK activation (30). In contrast to AR-KO, SBMA iMNs were hypophosphorylated at sites (Ser485/491 and Ser182) (Figure 4A), which are required for AMPK regulation and localization (31, 32). Activated AMPK inhibits mTORC1 signaling, which leads to hypophosphorylation of P70S6K and $4 \mathrm{E}-$ binding protein 1 (4E-BP1) and a reduction in protein synthesis. As expected, there was a decrease in phosphorylation of mTOR and 4E-BP1 in AR-KO, but hypophosphorylation was not detected in SBMA (Figure 4B). mTORC1 was hyperphosphorylated at Thr2446 and Ser2448 in SBMA cells, indicating an overall increased kinase activity (33). Consistently, 4E-BP1 showed increased phosphorylation at different residues in SBMA cells (Figure 4B). These data suggest that, in the case of cellular ATP deficiency, mTORC1 and consequently protein synthesis are reduced in AR-KO, while remaining active in SBMA.

The AMPK phosphorylation level was consistent with the phosphorylation level of myocyte enhancer factor-2 (MEF2), a direct downstream AMPK target involved in carbohydrate metabolism. MEF2 phosphorylation was reduced in SBMA and increased in AR-KO when compared with control (Figure 4C). AMPK-mediated phosphorylation of MEF2 increases GLUT4 expression and thereby increases glucose transport and the support of energetic demands in nerve terminals (34). Similarly, the sterol regulatory element-binding protein (SREBP-1), a transcription factor responsible for fatty acid metabolism (35), was hypophosphorylated in SBMA cells (Figure 4D). AMPK directly phosphorylates SREBP to control lipid homeostasis $(35,36)$. Low SREBP-1 phosphorylation in SBMA cells might be explained by the concomitant decrease in overall AMPK activity.

In response to low energy levels, heat shock factor 1 (HSF1), a key regulator of adaptive stress response, was hypophosphorylated in AR-KO cells (Figure 4D). Loss of phosphorylation at Ser303 sensitizes HSF1 activation under nutrient-deprived conditions (37). In contrast, SBMA cells did not show an increase or decrease in HSF1 phosphorylation. This implies an insufficient adaptive response to low energy and nutrient levels in SBMA iMNs.

In addition, calcium/calmodulin dependent protein kinase I (CaMK1), an upstream regulator of AMPK, was hyperphosphorylated at Thr177 in SBMA but not in AR-KO (Figure 4E). Thr177 and Thr196 are key phosphorylation sites for CaMK1 catalytic activity (38). Activation of CaMK1 may be part of a feedback mechanism in response to decreased AMPK signaling (39). Altogether, these results provide further evidence that bioenergetic adaptation is compromised in SBMA (Figure 4F).

Reduced Acetyl-CoA exacerbates mitochondrial impairment in SBMA. Given the large number of metabolic genes with diminished expression in SBMA iMNs, we asked whether alteration in these metabolic pathways impacts mitochondrial function. Acetyl-CoA is an intermediate metabolite and the product of multiple catabolic processes; it is also a substrate for anabolic reactions. It responds to homeostatic perturbations by controlling the equilibrium among metabolic reactions, as well as providing an energetic fuel for the tricarboxylic acid (TCA) cycle and oxidative phosphorylation. Furthermore, the genes 
A

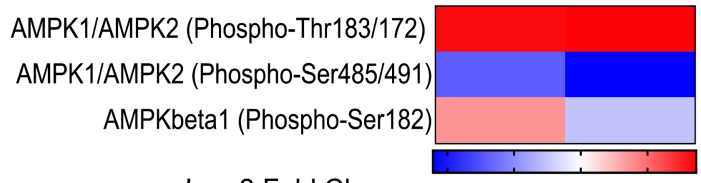

Log 2 Fold Change-0.4 $\begin{array}{llll}-0.2 & 0 & 0.2\end{array}$

B

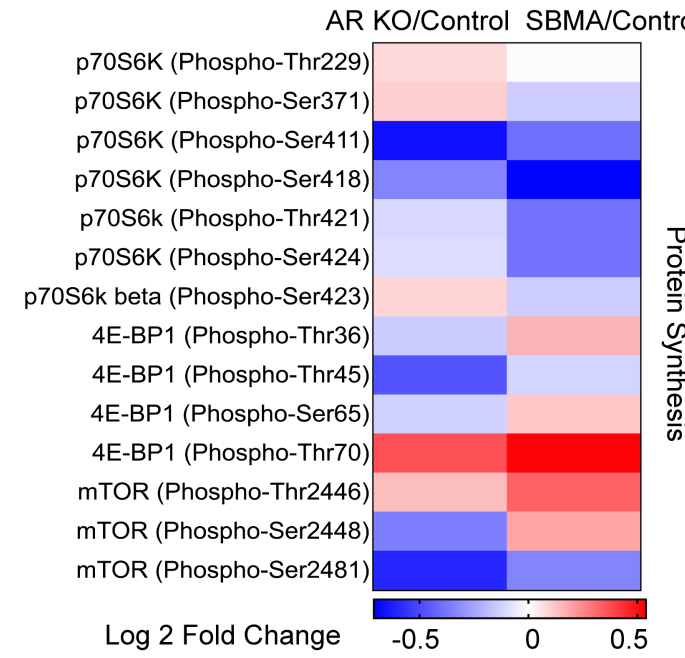

F
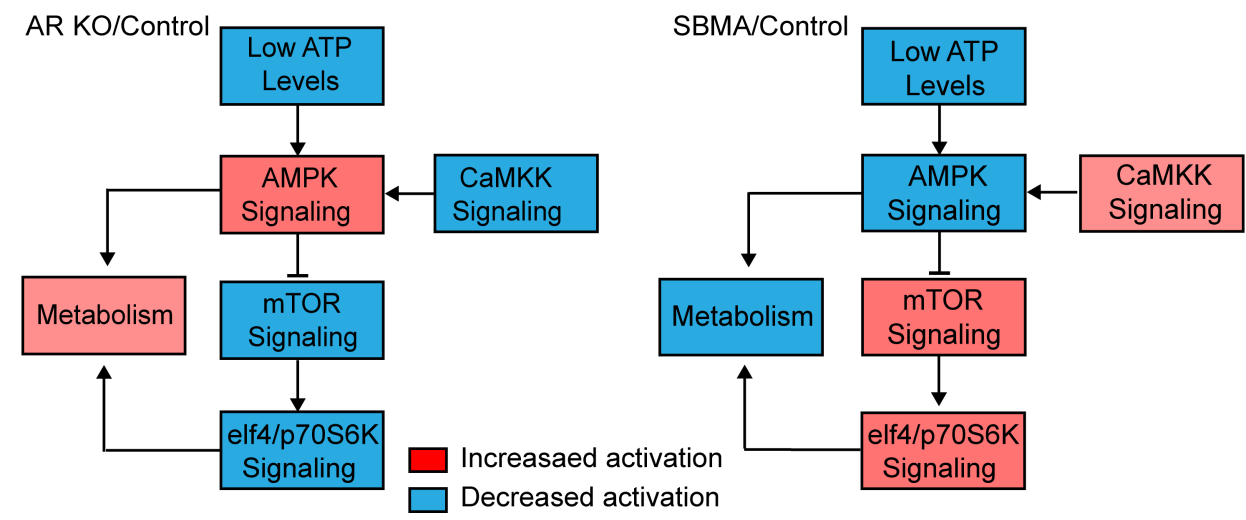

AR KO/Control SBMA/Control

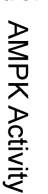

D

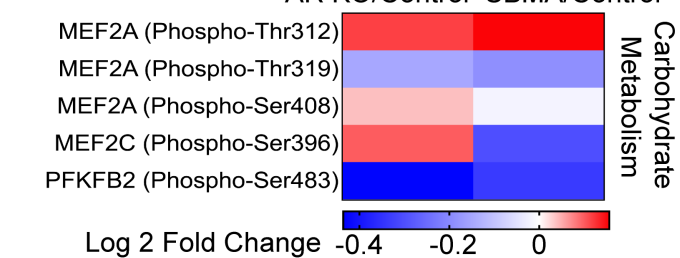
AR KO/Control SBMA/Control

ACC1 (Phospho-Ser79)

ACC1 (Phospho-Ser80)

HNF4a (Phospho-Ser313)

HSF1 (Phospho-Ser303)

HSL (Phospho-Ser552/563)

HSL (Phospho-Ser554)

SREBP-1 (Phospho-Ser439)

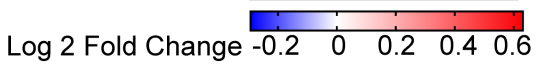

E

AR KO/Control SBMA/Control

CaMK1 alpha (Phospho-Thr177)

CaMK2 (Phospho-Thr286)

CaMK2 (Phospho-Thr287)

CaMK4 (Phospho-Thr196/200)

LKB1 (Phospho-Thr189)

LKB1 (Phospho-Ser428)

PKA CAT (Phospho-Thr197)

PLC-beta (Phospho-Ser1105)

PLC-beta3 (Phospho-Ser537)

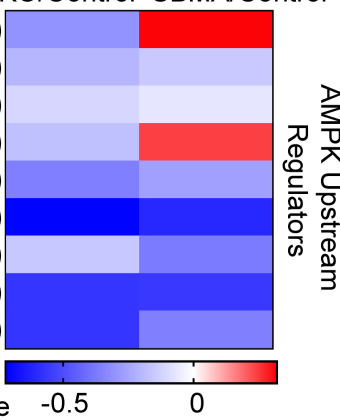

Log 2 Fold Change $\quad-0.5 \quad 0$

\section{둥.
$\frac{0}{0}$
$\frac{1}{3}$
$\frac{1}{10}$
$\frac{0}{0}$
$\frac{0}{0}$
3}

Figure 4. Reduced phosphorylation of proteins in the AMPK signaling pathway in SBMA iMNs. Heatmaps represents log fold changes (average ratio of signal intensity of phosphorylated antibody/average signal intensity of antibody). Signals were detected across 6 replicates of phosphorylation-specific antibodies and their nonphosphorylated pairs. (A) AMPK phosphorylation. (B) mTORC1 signaling and protein synthesis. (C) AMPK-mediated carbohydrate metabolism. (D) AMPK-mediated lipid metabolism. (E) AMPK upstream regulators. $N=3 \mathrm{SBMA}, N=3$ control, and $N=3$ AR-KO. (F) A simplified schematic of the AMPK signaling summarizing fold changes of SBMA/control relative to AR-KO/control. Proteins with SBMA/control and KO/control fold-change differences more than 1 SD (red) from the mean were identified as having overall increased activation (hyperphosphorylation). Fold-change differences below $1 \mathrm{SD}$ (blue) indicate an overall decreased activity (hypophosphorylation). Fold-change differences within $1 \mathrm{SD}$ of the mean indicates no difference between AR-KO/control and SBMA/control. iMNs were treated with $10 \mathrm{nM}$ DHT.

for key metabolic enzymes such as ATP citrate lyase (ACLY), the primary enzyme responsible for the synthesis of cytosolic acetyl-CoA; acyl-CoA synthases (ACSL1 and ACSL3), enzymes required for fatty acid $\beta$-oxidation; and branched-chain amino acid transaminase 2 (BCAT2), which catalyzes the first reaction in the breakdown of ketogenic amino acids, showed reduced H3K27ac levels near their TSS in SBMA cells (Figure 5A). This led us to measure the level of acetyl-CoA, which is the product of ketogenic amino acid and lipid breakdown. We hypothesized that, if these compensatory metabolic pathways are affected in SBMA, acetyl-CoA - as a central intermediate metabolite - would also be affected. 
We found significantly lower levels of intracellular acetyl-CoA in SBMA and also AR-KO iMNs (Figure 5B). Previous studies have shown that mitochondrial impairment occurs in SBMA (10).

To assess whether low concentrations of acetyl-CoA have functional consequences for respiratory chain efficiency, we restored intracellular acetyl-CoA levels in SBMA motor neurons and measured mitochondrial ATP production rate. To do this, we cultured cells in a medium supplemented with high sodium pyruvate concentrations (40) for 3 days before assessing mitochondrial function with the Seahorse assay. Once pyruvate enters the cell, it is converted into acetyl-CoA for chromatin acetylation and mitochondrial ATP production (Figure 5A). Increasing pyruvate concentration increased the oxygen consumption rate (OCR) in SBMA iMNs and, subsequently, the mitochondrial ATP production rate (Figure 5, C and D). To further assess whether perturbation in metabolic pathways such as low acetyl-CoA levels contributes to degeneration, we assessed neuronal cell death over time. Sodium pyruvate treatment significantly increased SBMA iMN survival at $8 \mathrm{dp}$ $(P<0.001$; Figure 5, F and G, and Supplemental Figure 6A). The SBMA cells appeared healthier, with less cellular aggregation detected compared with untreated cells (Supplemental Figure 6B). In parallel, inhibiting the electron transport chain (ETC) in control cells reduced intracellular acetyl-CoA levels (Figure 5E). A cocktail of 3 ETC inhibitors, rotenone (complex I), antimycin (complex III), and oligomycin (complex V), was used to mimic respiratory defects in control cells. After an 8-hour treatment of cultured control iMNs, the acetyl-CoA level decreased dramatically compared with untreated cells. After 24 hours of blocking the ETC complex, the decrease in acetyl-CoA was no longer significant (Figure 5E), perhaps because the levels were restored by compensatory metabolic processes. Furthermore, inspection of the mitochondria by electron microscopy showed a well-organized and dense matrix across all cell lines (Supplemental Figure 7A). Additionally, we examined the expression of ETC subunits that are labile when the complex is not assembled. A change in the levels of these proteins can be indicative of changes in complex assembly. Our results showed no change in components of the 5 ETC complexes in SBMA and AR-KO iMNs compared with controls (Supplemental Figure 7, B and C). This suggests that defects in SBMA oxidative phosphorylation are not associated with altered ETC complex integrity and further highlights the link between perturbation in metabolic processes and mitochondrial impairment. Taken together, these results suggest that decreased acetyl-CoA is a response to impairment in compensatory metabolic pathways, which exacerbates mitochondrial dysfunction in SBMA.

Reduced P300/CBP acetylation coincides with reduced intracellular acetyl-CoA levels. H3K27ac is a known target of the histone acetyltransferase (HAT) p300 cyclic AMP response element-binding protein (CBP) (41), and its acetylation is dynamically affected by intracellular acetyl-CoA concentration (Figure 5A) (42). HATs use acetyl-groups donated by acetyl-CoA in their acetyl transfer reaction (43). Western blot analysis demonstrated decreased levels of acetylated P300/CBP in SBMA iMNs (Supplemental Figure 6, C and D). Collectively, these data connect reduced H3K27 acetylation and impaired ETC function in SBMA iMNs through low levels of acetyl-CoA, a common substrate for the enzymatic activity of p300/CBP and ETC function (Figure 6).

\section{Discussion}

In this study, we integrated gene expression analysis with a genomic mapping of H3K27ac and AR to assess how alteration in histone acetylation influences the transcriptomic landscape in SBMA motor neurons. To investigate the relationship between mutant $\mathrm{AR}$ transcriptional dysregulation and other pathologic molecular processes, we derived motor neuron-like cells from human iPSCs. Through ChIP-seq and RNA-seq experiments in SBMA iMNs, we have shown that reduced histone H3K27 acetylation coincides with the repression of metabolic genes. These results are consistent with the well-known role of histone acetylation in regulating gene expression. In addition to $\mathrm{H} 3 \mathrm{~K} 27 \mathrm{ac}$, another histone acetylation marker, $\mathrm{H} 4 \mathrm{~K} 12 \mathrm{ac}$, is associated with gene expression (44). Similar to H3K27, H4K12 and other histones are acetylated by P300/CBP (45). Altogether, considering the low specificity of P300/CBP (46), H3K27ac may be just one of the markers and contributors to acetylation and reduced gene expression. Thus, in addition to H3K27ac, reduction of other acetylated histones may be contributing to the overall repression of metabolic genes in SBMA. Decreased H3K27ac in the striatum of $\mathrm{HD}$ transgenic mice (47) and general loss of H3K27ac has been shown in the brains of aging humans and mice (48). These data further validate the notion that genetic and epigenetic changes coparticipate in regulating gene expression in SBMA and perhaps other age-related neurodegenerative diseases.

We suggest that decreased $\mathrm{H} 3 \mathrm{~K} 27 \mathrm{ac}$ occurs as a result of reduced P300/CBP acetylation (45). Decreased histone acetylation may result from low intracellular acetyl-CoA levels $(49,50)$, and as a key metabolite, acetyl-CoA may link metabolism and chromatin remodeling. In light of the importance of acetyl-CoA production for mitochondrial function, we measured intracellular acetyl-CoA levels. Low acetyl-CoA levels and low 
A
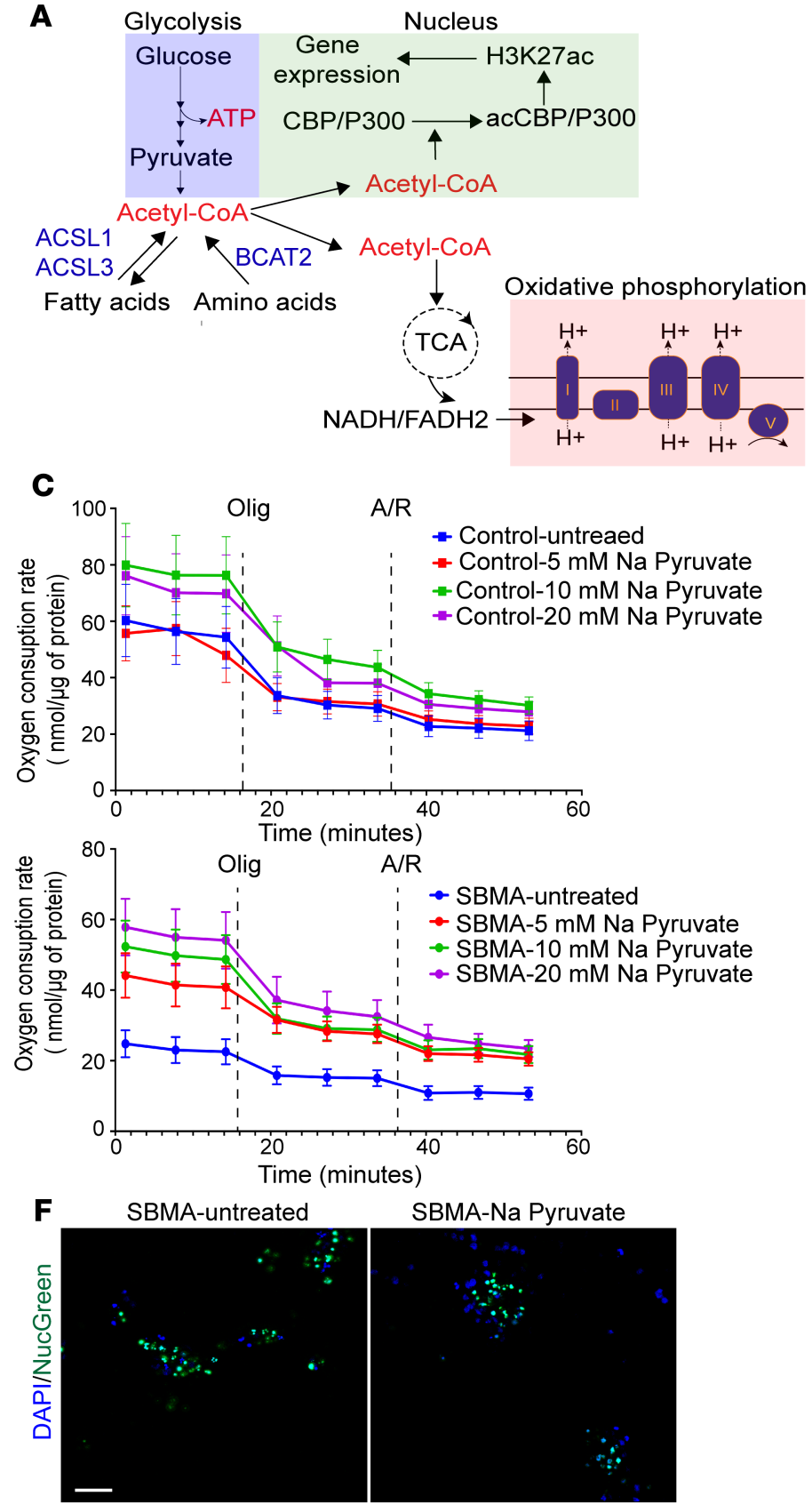

SBMA-Na Pyruvate

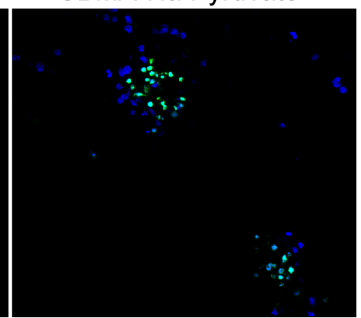

B
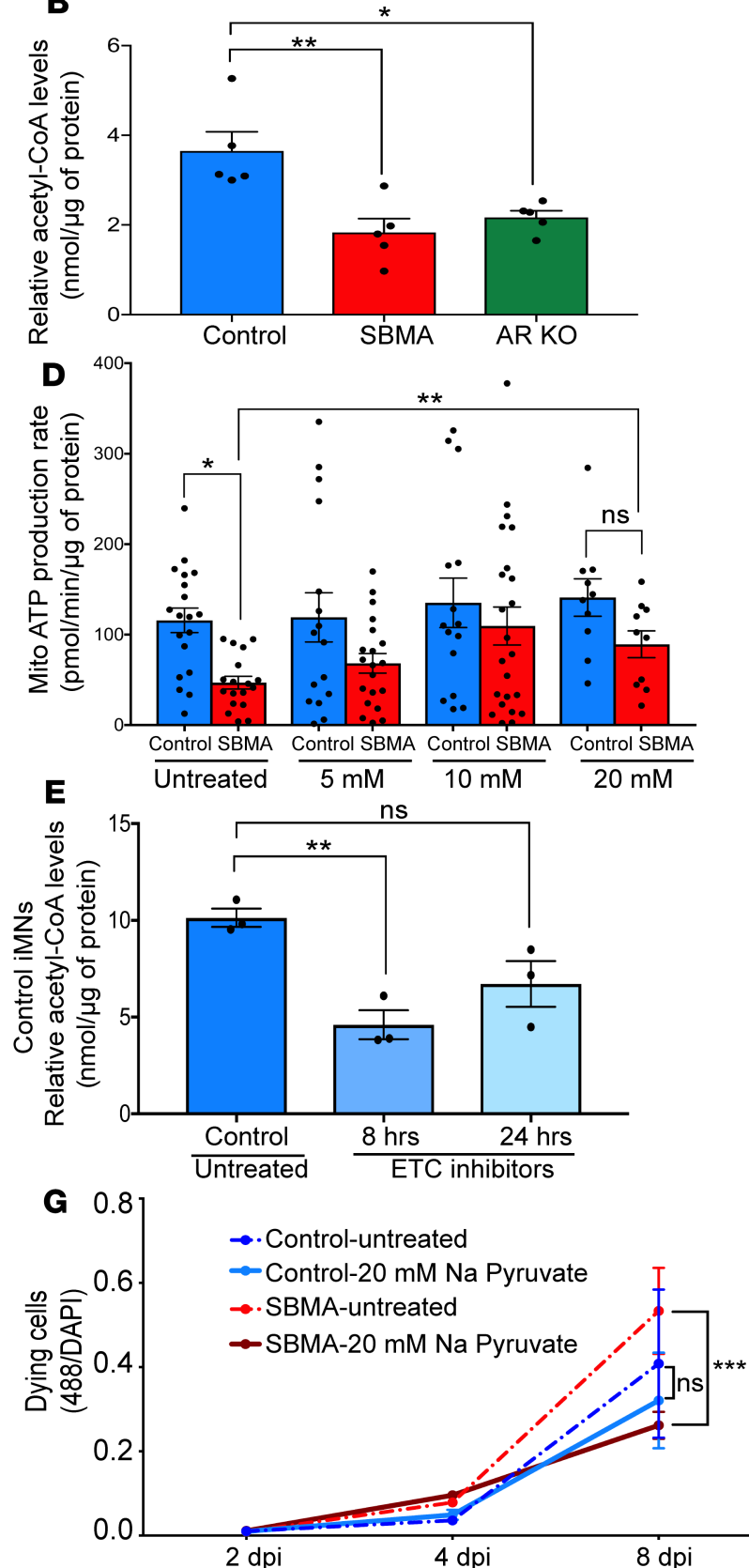

Figure 5. Sodium pyruvate treatment improves SBMA mitochondrial function and increases SBMA iMN survival. (A) A simplified metabolic flow diagram linking oxidative phosphorylation and histone acetylation. ACSL1, ACSL3, and BCAT2 are genes with greater than 2-fold reduced H3K27ac identified from the ChIP-seq data (SBMA versus control). (B) Relative acetyl-CoA levels. iMNs were grown in triplicate wells from the same batch of differentiation. (C and D) Bioenergetic extracellular flux analysis on iMNs treated with $20 \mathrm{mM}$ sodium pyruvate from 2-6 dpi, every 2 days. (C) OCR in control (top) and SBMA (bottom) after 48-hour treatment with sodium pyruvate. Oligomycin, or antimycin/rotenone (A/R) treatments were given at the indicated time points. (D) Rate of mitochondrial ATP production in iMNs treated with sodium pyruvate. Cells were grown in triplicate wells per cell line/per condition. Error bars show mean $\pm \mathrm{SE} ;{ }^{*} P<0.05$, ${ }^{* *} P<0.01$, 2-tailed Student's $t$ tests. (E) Relative acetyl-CoA levels in control iMNs treated with DMSO and a mix of ETC inhibitor cocktail (1.5 $\mu \mathrm{M}$ oligomycin, $0.5 \mu \mathrm{M}$ of each antimycin and rotenone). iMNs were treated for 8 hours or 24 hours. Cells were grown in triplicate wells from the same batch of differentiation. (F and $\mathbf{G}$ ) Real-time cell viability assessment of iMNs treated with 20 mM sodium pyruvate using NucGreen dead 488. (F) Representative images of SBMA iMNs treated with sodium pyruvate. NucGreen dead 488 (Green) and DAPI (blue). Scale bar: $50 \mu \mathrm{m}$. (G) For quantification, 4 images per cell line were taken at each time point. Error bars show mean $\pm \mathrm{SE}$; ${ }^{* *} P<0.001$, 2-tailed Student's $t$ tests. All experiments were performed on $N=3 \mathrm{SBMA}, N=3$ control, and $N=3 \mathrm{AR}-\mathrm{KO}$. Error bars show mean $\pm \mathrm{SE}$; ${ }^{*} P<0.05$ and ${ }^{* *} P<0.01$; 1-way ANOVA followed by Bonferroni's multiple comparisons test, unless otherwise indicated. iMNs were treated with $10 \mathrm{nM}$ DHT. 


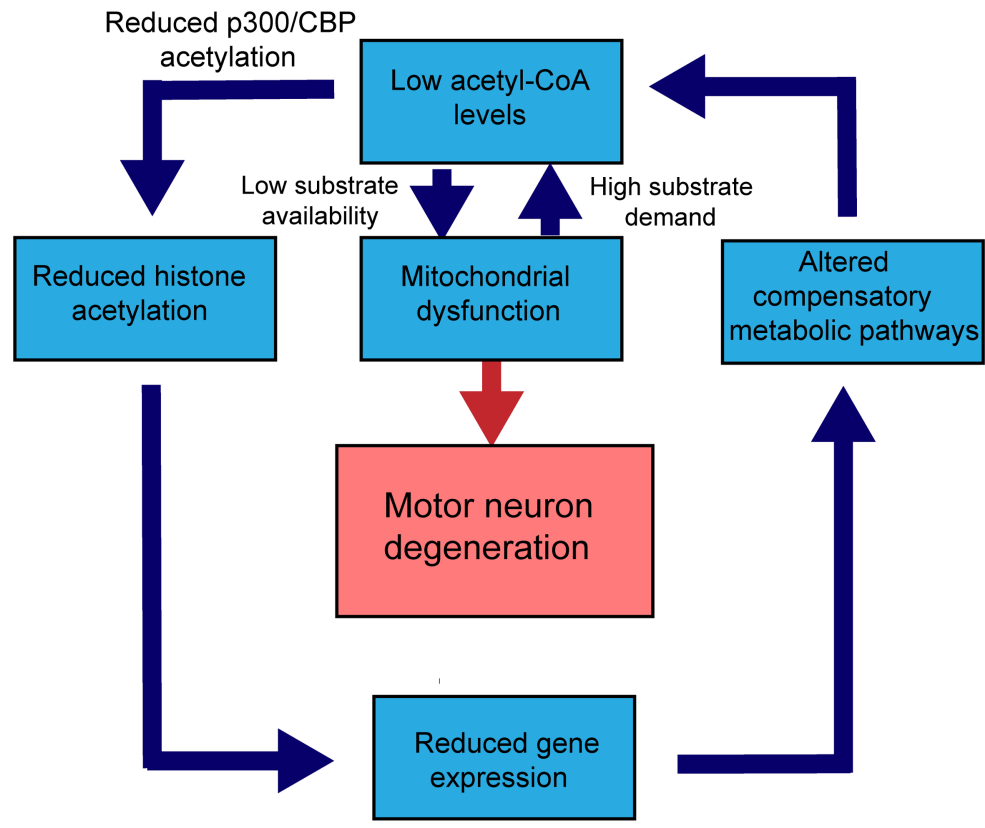

Figure 6. The interrelationship between mitochondrial dysfunction and transcriptional dysregulation. Flow diagram depicting low acetyl-CoA levels as a central metabolic change that connects reduced expression of metabolic genes to mitochondrial ATP production impairment. From left, altered p300/CBP activity, which may be due to sequestration and depletion by the mutant AR (40), leads to reduced H3K27ac. This in turn contributes to repression of ETC and metabolic genes. Repression of these genes perturbs compensatory metabolic pathways, which results in insufficient production of acetyl-CoA. Acetyl-CoA is a common substrate for both the ETC function and p300/CBP HAT activity. Low acetyl-CoA (substrate) contributes to low ATP production and mitochondrial dysfunction. In parallel, when the energy demands of the cell increase, mitochondria respond by consuming more acetyl-CoA (substrate). The reduction of acetyl-CoA contributes to repression of ETC and metabolic genes through impaired p300/CBP acetylation.

mitochondrial ATP production in SBMA iMNs suggest 2 nonmutually exclusive possibilities: an uncharacterized integral impairment in mitochondrial function that increases mitochondrial demand for acetyl-CoA to sustain bioenergetic needs, or an inability of metabolic pathways to synthesize sufficient acetyl-CoA levels for mitochondrial function. Acetyl-CoA fuels the mitochondrial TCA cycle and the ETC for ATP production through oxidative phosphorylation, and it also acts as a substrate for HATs such as P300/CBP to acetylate histones (51). Restoring intracellular acetyl-CoA levels with pyruvate increased mitochondrial ATP production rate in SBMA, indicating that the mitochondrial function can improve if resources are available. On the other hand, blocking the ETC in control iMNs reduced acetyl-CoA levels. Under energy deprivation, such as blocking the ETC function, mitochondria restore bioenergetic homeostasis by consuming more acetyl-CoA. In parallel, metabolic pathways produce the resources for mitochondria to maximize ATP production. This was evident when acetyl-CoA levels increased after prolonged ETC inhibition in control cells, highlighting the role of compensatory metabolic pathways. We and others (17) have shown that motor neurons are predominantly oxidative and, therefore, have a low glycolytic profile. This suggests that control iMNs depend on other compensatory pathways such as protein and lipid breakdown to restore acetyl-CoA levels with prolonged ETC inhibition. These changes are accompanied by evidence of decreased expression of genes regulating compensatory metabolic pathways and inadequate AMPK signaling in response to low ATP levels. Alterations in metabolic gene expression have also been reported in the skeletal muscle of AR113Q SBMA mice (12). Similar to motor neurons, the skeletal muscle demands high levels of energy. Impaired mitochondrial function is a recurrent theme in SBMA and other polyglutamine expansion disease $(7,9,12,52,53)$. Collectively, our results suggest that depletion of ATP in SBMA iMNs is indicative of insufficient substrate due to perturbations in compensatory metabolic pathways.

The changes that we reported in this study suggest that alteration in metabolic pathways and impairment in mitochondrial function arise due to both loss of function and gain of function of the mutant AR in SBMA iMNs. However, these changes are less marked in AR-KO iMNs, where ATP levels are more stable and where cell survival is not significantly impaired. Therefore, mutant AR toxic gain of function does more to perturb energy homeostasis than does loss of function. 
Our findings do not exclude the possibility that mutant AR can directly damage mitochondrial function (10). Rather, we add to this notion by introducing metabolic adaptation failure as a contributor to this impairment. Both processes could result in mitochondrial stress. The accumulation of these insults results in vulnerability to cells with pathogenic polyglutamine repeat expansion (54), consistent with our interpretation that the disease cells are unable to properly compensate and adapt to these insults.

Overall, our study indicates a molecular link between mitochondrial function, metabolism and epigenetic regulation that may be important for motor neuron survival. Our analysis suggests that SBMA motor neurons are sensitive to insufficient metabolic adaptation and its exacerbation of mitochondrial dysfunction, which then leads to neurodegeneration. We propose that epigenetic dysregulation of the metabolic genes contributes to impairment of mitochondrial ATP production through perturbation of compensatory metabolic pathways and insufficient substrate production in response to disrupted cellular energy homeostasis.

The reversibility of this process provides opportunities for therapeutic intervention through metabolic pathways that impact mitochondrial function in SBMA and perhaps other neurodegenerative diseases.

\section{Methods}

\section{hNIL transfection of human iPSCs}

iPSCs were transfected with the hNIL construct as previously described (15). Briefly, iPSCs were grown to $80 \%$ confluence and were then digested with accutase solution into single cells. Cells were passaged on Matrigel-coated plates in E8 Flex media supplemented with $10 \mu \mathrm{M}$ ROCK inhibitor (Tocris). A total of 3 $\mu \mathrm{g}$ of total DNA (hNIL donor construct and each of the site- specific TALEN or CRISPER/cas9) was added using Lipofectamine Stem (Thermo Fisher Scientific). Forty-eight hours later, transfected iPSCs were dissociated with accutase and replated sparsely onto Matrigel-coated dishes in E8 flex and $10 \mu \mathrm{M}$ ROCK inhibitor. Individual clones were manually picked and karyotyped.

\section{Motor neuron differentiation from human iPSCs}

Differentiation was performed as previously described (15). In brief, stably transfected iPSCs with the hNIL cassette were plated at $30,000 \mathrm{cells} / \mathrm{cm}^{2}$ on a Matrigel-coated surface in E8 Flex medium (Thermo Fisher Scientific) containing $10 \mu \mathrm{M}$ ROCK inhibitor. Twenty-four hours later, cells were induced with Neural Induction Medium (NIM): DMEM/F12, B27 (1×), N2 (1×), NEAA (1×), GlutaMax $(1 \times)$, compound $\mathrm{E}(0.2 \mu \mathrm{g} / \mathrm{mL}$; Calbiochem), ROCK inhibitor (10 $\mu \mathrm{M}$; Tocris), doxycycline $(2 \mu \mathrm{g} / \mathrm{mL}$; Clontech), and DHT (10 nM; MilliporeSigma). After 48 hours of doxycycline treatment, motor neurons were dissociated with accutase to single cells and replated 50,000 cells/ $\mathrm{cm}^{2}$ on PDL/ laminin-coated surfaces in Neural Differentiation Medium (NDM): DMEM/F12:BrainPhys neuronal medium, B27 $(1 \times)$, N2 $(1 \times)$, NEAA $(1 \times)$, GlutaMax $(1 \times)$, compound E $(0.2 \mu \mathrm{g} / \mathrm{mL})$, ROCK inhibitor $(10 \mu \mathrm{M})$, doxycycline $(2 \mu \mathrm{g} / \mathrm{mL})$, laminin $(1 \mu \mathrm{g} / \mathrm{mL}$; Roche), BDNF (10 ng/mL; Peprotech), GDNF (10 ng/mL; Peprotech), NT3 (10 ng/mL; Peprotech), and DHT (10 nM; MilliporeSigma). On day 4 onward, half of cell culture medium was removed and replaced by Neuron medium (NM): BrainPhys neuronal medium, B27 (1×), N2 (1×), NEAA $(1 \times)$, GlutaMax $(1 \times)$, compound E $(0.2 \mu \mathrm{g} / \mathrm{mL})$, ROCK inhibitor $(10 \mu \mathrm{M})$, doxycycline $(2 \mu \mathrm{g} / \mathrm{mL})$, laminin $(1 \mu \mathrm{g} / \mathrm{mL})$, BDNF $(10 \mathrm{ng} / \mathrm{mL})$, GDNF $(10 \mathrm{ng} /$ $\mathrm{mL})$, NT3 (10 ng/mL), and DHT (10 nM). iPSCs were treated with $10 \mathrm{nM} \mathrm{DHT}$ on first day with doxycycline induction, and every 48 hours thereafter. Reagents are from Thermo Fisher Scientific except where indicated.

\section{RNA-seq sample preparation}

Trizol/Chloroform total RNA extraction was performed, followed by QIAGEN RNeasy Mini Kit column purification. RNA integrity number (RIN) was verified using an Agilent Bioanalyzer. Sequencing libraries were constructed from $\sim 1 \mu \mathrm{g}$ total RNA using TruSeq Stranded Total RNA Library Prep Gold (Illumina), used according to manufacturer's instructions except where noted. Adapters were Illumina 6-base single indexed. Amplification was performed using 10 cycles, which was optimized for the input amount and to minimize the chance of overamplification. Libraries were pooled in equimolar amounts for sequencing. The pooled libraries were sequenced on a HiSeq 4000 to achieve a minimum of 100 million 75 base read pairs. The data were processed using RTA version 2.7.7. 


\section{ChIP sample preparation}

For IP of H3K27ac (Abcam, ab4729), H3K4me1 (MilliporeSigma, 07-436), H3K4me3 (Abcam, ab8550), and RNA Pol II (Abcam, ab5095), $20 \times 10^{6}$ iMNs were used; for AR IP (Santa Cruz Biotechnology Inc., N20), $100 \times 10^{6}$ iMNs were used. iMNs were treated with $1 \%$ formaldehyde for 10 minutes in $37^{\circ} \mathrm{C}$, followed by $0.125 \mathrm{M}$ glycine in PBS. Cells were then washed 2 times with cold PBS and scraped in PBS. Cells were then centrifuged at $1500 \mathrm{~g}$ for 5 minutes at $4^{\circ} \mathrm{C}$. A total of 20 $\times 10^{6}$ iMNs was resuspended in RIPA buffer $(10 \mathrm{mM}$ Tris-HCl, $\mathrm{pH} 7.6,1 \mathrm{mM}$ EDTA, $0.1 \%$ SDS, $0.1 \%$ Na-deoxychyolate, $1 \%$ Triton X-100) supplemented with EDTA-free protease inhibitor (Roche) Cells were sonicated using TC12×24 tube on Covaris S2, duty cycle $20 \%$, intensity 5 , cycle/burst 200 , 60 -second cycles $(\times 30$ cycles). Chromatin size was evaluated on a $2 \%$ gel with a target size of $200-500$ bp. Samples were centrifuged at 20,000 $\mathrm{g}$ for 10 minutes at $4^{\circ} \mathrm{C}$. For IP, $40 \mu \mathrm{L}$ Dynabeads Protein A was incubated with $10 \mu \mathrm{g}$ ChIP antibody for 45 minutes at room temperature (RT) on a shaker. In parallel, $40 \mu \mathrm{L}$ Dynabeads Protein A was incubated with $10 \times 10^{6}$ cells for 30 minutes at $4^{\circ} \mathrm{C}$ to preclear chromatin on a tube rotator. Next, beads were washed 2 times with PBS for 5 minutes each time in RT. The precleared chromatin was transferred into the antibody-bound beads and incubated on a tube rotator overnight in $4^{\circ} \mathrm{C}$. following overnight incubation, samples were washed for 10 minutes with rotation at $4^{\circ} \mathrm{C}$ with $2 \times$ RIPA buffer, $2 \times \mathrm{RIPA}+0.3 \mathrm{M} \mathrm{NaCl}, 2 \times \mathrm{LiCl}$ buffer $(0.25 \mathrm{M} \mathrm{LiCl}, 0.5 \%$ NP-40, $0.5 \%$ NaDOC), $1 \times \mathrm{TE}(\mathrm{pH} 8)$ plus $0.2 \%$ Triton X-100 and $1 \times \mathrm{TE}(\mathrm{pH} 8)$. After the last wash, beads were resuspended with TE (pH8) $+10 \%$ SDS supplemented with $20 \mathrm{mg} / \mathrm{mL}$ proteinase $\mathrm{K}$ and incubated at $65^{\circ} \mathrm{C}$ for 4 hours in a shaker. The supernatant was collected using a magnet, and DNA was purified using phenol/chloroform/isoamyl alcohol. The DNA concentration was measured using Pico-Green dsDNA assay kit (Thermo Fisher Scientific). Chip-seq libraries were constructed from 10 ng of ChIP DNA using Ovation Ultralow System V2 1-96 using 15 cycles of PCR amplification. The final libraries were twice purified using Ampure XP PCR Purification Beads (Agencourt). The libraries were pooled and then quantitated by qPCR. The pool balance was checked by performing a MiSeq run of 25 cycles plus index read using a MiSeq Nano kit, version 2. The percentage of each library in the pool was determined from the demultiplexing and was used to rebalance the pool before sequencing. The pooled libraries were sequenced on multiple lanes of a HiSeq 2500 in Rapid mode using version 3 chemistry to achieve a minimum of 18 million 51-base reads. The data were processed using RTA version 1.18.66.3 and CASAVA 1.8.2.

\section{Extracellular flux analysis}

For Seahorse analysis (XF96, Agilent Technologies), iMNs were seeded at a density of $5 \times 10^{4}$ cells/well in XF96 cell culture microplates. Two dpi and 4 dpi iMNs were tested 8 hours and 48 hours after replating cells, respectively. Cells were simultaneously tested for OCR and extracellular acidification rate (ECAR) following the manufacturer's instructions with the injection of Seahorse XF Real-Time ATP Rate Assay Kit (Agilent Technologies). Data were normalized to total cellular protein by lysing the iMNs in RIPA buffer (50 mM Tris, $0.1 \%$ SDS, $0.5 \%$ sodium deoxycholate, $1 \%$ Triton X-100, $150 \mathrm{mM} \mathrm{NaCl}$ ), supplemented with $1 \times$ phosphatase and protease inhibitor cocktail (Roche). Total cellular protein was then quantified via BCA assay (Thermo Fisher Scientific). For rescue assay using sodium pyruvate, iMNs were treated with 5 , 10, and $20 \mathrm{mM}$ sodium pyruvate (Thermo Fisher Scientific) from 2 dpi every day until the day of the assay (4 dpi). Buffer factor was calculated for each cell culture medium with different sodium pyruvate concentrations using the Seahorse XF buffer factor protocol.

\section{ATP biosensor (GoATEAM)}

IPSCs were transduced with lentivirus vector with the GoATeam plasmid (gift from Kelly A. Chamberlain, laboratory of Zu-Hang Sheng, NINDS, NIH, Bethesda, MD; developed by Hiroyuki Noji, University of Tokyo, and Hiromi Imamura, Kyoto University, Japan). iMNs were replated on a PDL/ laminin-coated glass-bottom dish on $2 \mathrm{dpi}$. Cells for imaging were maintained on a microscope at $37^{\circ} \mathrm{C}$ with a continuous supply of $95 \%$ air and $5 \%$ carbon dioxide mixture using a stage-top incubator. iMNs were transduced with lentivirus 4 days before taking the first image. Plates were seeded at the same density, and live images were taken from the same plate over time. Comparison of orange fluorescent protein (OFP)/green fluorescent protein (GFP) emission ratio of GoATEAM at different time points was calculated from fluorescent images using ImageJ (NIH). 
Cell viability assay

Cell viability was measured using NucGreen Dead 488 Ready probes Reagent (Thermo Fisher Scientific), as per manufacturer's instructions. Cells were seeded after 48 hours of doxycycline treatment at a density of $5 \times 10^{4}$ cells/well in 96-well plates and were imaged using a Nikon Biostation CT on 2,4,8, and 10 dpi. Image analysis of NucGreen signal was automated and performed with custom MATLAB scripts. Briefly, background signal was subtracted from each channel, cells were segmented by intensity and then size, and fluorescence intensity was measured within cells. Fluorescence intensity was normalized to DAPI signal to account for cell number. For single-cell analysis, whole cells were identified with mCherry signal and nuclei with DAPI signal. Nuclei were segmented from each other and then used to separate cells with iterative water shedding algorithms before calculating fluorescence intensity for each channel. Cells were then filtered by the average DAPI intensity to remove debris.

\section{AMPK signaling phospho-antibody array}

Phosphorylation of proteins involved in AMPK signaling pathway were examined using the Full Moon BioSystems Antibody Microarray (Full Moon BioSystems Inc.), following the manufacturer's instructions. Briefly, protein samples from 6 dpi iMNs were biotinylated, and 80 OD of lysate sample was incubated with antibody array. Signals were detected using $0.5 \mathrm{mg} / \mathrm{mL}$ Cy3-Streptavidin (Invitrogen). Data analysis was performed by extracting the median signal intensity of each spot on the array. Average signal intensity of replicate spots was normalized to median signal. Signal ratio of the paired antibodies was determined using the average signal intensity of replicate spots, for each pair of site-specific antibody and phospho-site specific antibody.

\section{Acetyl-CoA measurement}

Intracellular acetyl-CoA levels were detected using Acetyl CoA Assay Kit (Biovision), following the manufacturer's instructions. Total cellular protein was quantified by BCA assay (Pierce; Thermo Fisher Scientific). For ETC inhibition, iMNs were treated with $1.5 \mu \mathrm{M}$ oligomycin and $0.5 \mu \mathrm{M}$ of each antimycin and rotenone for 8 or 24 hours.

Cell viability assay

Cell viability was measured using NucGreen Dead 488 Ready probes Reagent (Thermo Fisher Scientific), per manufacturer's instructions. Cells were seeded after 48 hours of doxycycline treatment at a density of $5 \times 10^{4}$ cells/well in 96-well plates and were imaged using a Nikon Biostation CT on 2,4,8, and 10 dpi. Image analysis of NucGreen signal was automated and performed with custom MATLAB scripts. Briefly, background signal was subtracted from each channel, cells were segmented by intensity and then size, and fluorescence intensity was measured within cells. Fluorescence intensity was normalized to DAPI signal to account for cell number. For single-cell analysis, whole cells were identified with mCherry signal and nuclei with DAPI signal. Nuclei were segmented from each other and then used to separate cells with iterative water shedding algorithms before calculating fluorescence intensity for each channel. Cells were then filtered by the average DAPI intensity to remove debris.

\section{Subcellular protein fractionation}

Isolation of total, cytoplasmic, and nuclear protein extracts was carried out using NE-PER (Thermo Fisher Scientific), following manufacturer's instructions. In brief, $4 \times 10^{6} \mathrm{iMNs}$ were washed and scraped in cold cytoplasmic extracting cell lysis buffer. Next, cell lysate was incubated on ice for 10 minutes, followed by centrifugation at $16,000 \mathrm{~g}$ for 5 minutes. The supernatant (cytoplasmic fraction) was collected, and the pellet was washed 2 times with cold PBS. The pellet was resuspended in nuclear extraction buffer and incubated on ice for 1 hour with subsequent vortex on high setting. The sample was spun at $16,000 \mathrm{~g}$ for 5 minutes to collect the nuclear fraction.

\section{Protein isolation and immunoblotting analysis}

Cells were lysed with RIPA buffer supplemented with (50 mM Tris, 0.1\% SDS, 0.5\% sodium deoxycholate, $1 \%$ Triton $\mathrm{X}-100,150 \mathrm{mM} \mathrm{NaCl}$ ), supplemented with $1 \times$ phosphatase and protease inhibitor cocktail (Roche).The lysates were sonicated and centrifuged at $15,700 \mathrm{~g}$ for 10 minutes at $4^{\circ} \mathrm{C}$. Cell lysates were denatured at $95^{\circ} \mathrm{C}$ in $4 \times$ sample buffer $(60 \mathrm{mmol} / \mathrm{L}$ Tris, $\mathrm{pH} 6.8,2 \% \mathrm{SDS}, 25 \%$ glycerol, $0.1 \%$ bromophenol 
blue, $20 \% \beta$-mercaptoethanol), processed for 10\% SDS-polyacrylamide gel electrophoresis (SDS-PAGE), and electro-transferred to polyvinylidene fluoride membrane (MilliporeSigma). Membranes were probed with 1:1000 rabbit anti-AR (Santa Cruz Biotechnology Inc., sc-13062), 1:5000 mouse anti- $\alpha$-tubulin (MilliporeSigma, T6199), 1:1000 mouse anti-histone H1 (Thermo Fisher Scientific, PA5-30055), 1:1000 rabbit anti-ac-P300/CBP (Cell Signaling Technology, 4771), 1:1000 rabbit anti-P300 (Cell Signaling Technology, 86377), 1:1000 mouse anti-HDAC1 (Cell Signaling Technology, 5356), 1;1000 rabbit anti-H3 (MilliporeSigma, 05-928), 1:1000 rabbit anti-cleaved caspase-3 (Cell Signaling Technology, 9661), 1:1000 rabbit anticaspase-3 (Cell Signaling Technology, 9662), 1:5000 mouse anti-actin (MilliporeSigma, A2228), and 1:1000 rabbit anti-vinculin (Cell Signaling Technology, 13901). For ETC subunit detection, 1:1000 Total OXPHOS antibody cocktail (Abcam, ab110411) was added, and cell lysates were denatured in $4 \times$ sample buffer without further denaturation at $95^{\circ} \mathrm{C}$. All antibodies were diluted in Tris-buffered saline and $5 \%$ (weight/vol) nonfat dry milk. Membranes were incubated overnight at $4^{\circ} \mathrm{C}$. Immunoreactivity was detected using peroxidase-conjugated AffiniPure goat anti-rabbit or anti-mouse IgG (1:5000; Jackson ImmunoResearch) and visualized using chemiluminescence reagent (GE Healthcare) following the manufacturer's instructions. Western blots were quantified using the ImageJ software package. Statistical significance of differences between control and experimental values was determined using 1-way ANOVA or 2-tailed Student's $t$ test, where indicated. All data are reported as means \pm SEM, and statistical significance is indicated by asterisks. $P$ values of less than 0.05 were considered significant.

\section{Immunofluorescent staining}

For immunofluorescence analysis, samples were fixed for 10 minutes at RT with $4 \%$ paraformaldehyde and were subsequently washed 2 times with PBS. Next, cells were permeabilized with $0.2 \%$ Triton X-100 (1× PBS) for 10 minutes at RT, followed by blocking with 10\% BSA in $0.1 \%$ Triton X-100 and $0.1 \%$ Tween (PBST) for 1 hour at RT. Samples were then incubated overnight at $4^{\circ} \mathrm{C}$ with primary antibodies: 1:200 mouse anti-HB9 (DSHB, 81.5C10), 1:250 rabbit anti-ISL1 (Abcam, ab109517), 1:1000 rabbit anti-AR (Santa Cruz Biotechnology Inc., sc-13062), 1:1000 mouse anti- $\beta$ III tubulin (Abcam, ab78078), and1:1000 rabbit anti-cleaved caspase-3 (Cell Signaling Technology, 9661) in 3\% BSA in PBST. After overnight incubation, samples were washed 3 times with $0.1 \%$ Triton X-100 and incubated with goat anti-mouse and anti-rabbit fluorescent secondary antibody (Thermo Fisher Scientific) in 3\% BSA in PBST for 1 hour at RT. Next, slides were washed 3 times with $0.1 \%$ Triton X-100 and once with PBS at RT. The slides were mounted using ProLong Diamond Antifade Mountant with DAPI (Thermo Fisher Scientific). Images were captured using a Lecia TCS SP5 II confocal microscope.

\section{Electron microscopy}

iMNs were fixed with $4 \%$ glutaraldehyde in $0.1 \mathrm{~N}$ cacodylate buffer at $\mathrm{pH} 7.4$ for 30 minutes at RT, followed by an overnight incubation at $4^{\circ} \mathrm{C}$. Next, samples were washed in buffer, treated with $1 \%$ osmium tetroxide in $0.1 \mathrm{~N}$ cacodylate buffer ( $\mathrm{pH}$ 7.4) for 1 hour on ice. After wash, samples were en bloc stained with $1 \%$ uranyl acetate in $0.1 \mathrm{~N}$ acetate buffer ( $\mathrm{pH}$ 5.0) overnight at $4^{\circ} \mathrm{C}$, followed by dehydration with a series of graded ethanol and embedded in epoxy resins. Sections of 70-90 nm thickness were counterstained with uranyl acetate and lead citrate. Images were taken with a bottom-mounted digital CCD camera (AMT XR-100, Danvers, Massachusetts, USA).

\section{TUNEL assay}

TUNEL was performed using Click-iT TUNEL Alexa Fluor 488 Imaging Assay for microscopy according to the manufacturer's instructions (Invitrogen).

\section{Electrophysiology}

Whole-cell patch clamp recordings were obtained from iMNs at $28 \mathrm{dpi}$. All recordings were performed at physiological temperature $\left(32^{\circ} \mathrm{C}-34^{\circ} \mathrm{C}\right)$. Electrodes were pulled from borosilicate glass (World Precision Instruments) to a resistance of 4-8 M $\Omega$ using a vertical pipette puller (Narishige, PC-10). Whole-cell recordings were made using a Multiclamp 700B amplifier (Molecular Devices), filtered at $3 \mathrm{kHz}$ (Bessel filter), and digitized at $20 \mathrm{kHz}$ (Molecular Devices). Recordings were not corrected for a liquid junction potential. Uncompensated series resistance ranges from 10-30 M $\Omega$. In current-clamp mode, cells were biased to a membrane potential of $-70 \mathrm{mV}$; in voltage-clamp mode, a holding potential of $-70 \mathrm{mV}$ was 
applied. Internal solution consisted of (in mM): $130 \mathrm{~K}$-gluconate, $5 \mathrm{KCl}, 3 \mathrm{MgCl}, 2 \mathrm{Na} 2 \mathrm{ATP}, 0.3 \mathrm{NaGTP}$, 10 HEPES, 0.6 EGTA, with a calculated $\mathrm{ECl}$ of $-67 \mathrm{mV}$. To measure intrinsic properties (input resistance, membrane time constant, and excitability) current steps ( $800 \mathrm{~ms},-15$ to $50 \mathrm{pA}, 5-10 \mathrm{pA} / \mathrm{step}$ ) were injected. Parameters of the first action potential discharged were analyzed.

\section{Quantification and statistical analysis}

$R N A$-seq data analysis. Paired-end sequence files (.fastq) per sample were quality inspected using the FastQC tool and were then adaptor clipped (TruSeq3-PE-2.fa:2:30:10) and trimmed to remove 5' nucleotide bias (HEADCROP:12) and low quality calls (TRAILING:20 SLIDINGWINDOW:4:20 MINLEN:15) using the Trimmomatic tool. Surviving intact pairs of reads per sample were then imported into the CLCbio Genomics Workbench and reference mapped by sample in stranded fashion against the current instance of the human genome (GRCh38.82) using the "RNA-Seq Analysis" tool supported therein under default parameters (v11). Expression per known annotated gene (Homo_sapiens.GRCh38.82.chr.gtf) in transcripts per kilobase million (TPM) units were then exported from the Workbench and imported into R. In R, TPM expression per sample was pedestalled by 2 and then $\log _{2}$ transformed. Genes not having an expression value $>1$ after transformation for at least 1 sample were discarded as not detected, while expression across samples for genes not discarded were quantile normalized. To remove noise-biased expression values, locally weighted scatter plot smoothing (LOWESS) was applied across normalized expression for all genes by sample class (coefficient of variation by mean expression). LOWESS fits were then over-plotted and inspected to identify the common low-end expression value where the relationship between mean expression (i.e., "signal") and coefficient of variation (i.e., "noise") grossly deviated from linearity. Expression values were then floored to equal this value if less, while expression for genes not observed greater than this value for at least 1 sample were discarded as noise biased. For genes not discarded, expression differences across sample classes were tested for using the 1-way ANOVA test under Benjamini-Hochberg (BH) FDR multiple comparison correction (MCC) condition using sample class as the factor. Genes having Type III corrected $P<0.05$ by this test were then subset, and the Tukey's HSD post hoc test used to generate mean differences and $P$ values for each possible pairwise comparison of classes. Genes having a post hoc $P<0.05$ for a specific comparison and a linear difference of means $\geq 1.5 \times$ for the same comparison were deemed to have expression significantly different between the compared classes, respectively. Analysis of differentially expressed genes to identify significantly enriched functional categories was performed using IPA (QIAGEN).

\section{ChIP-seq peak calling and analysis}

ChIP-seq analysis. Sequence files (.fastq) per sample were quality inspected using the FastQC tool and were then adaptor clipped and quality trimmed depending on being single-end (TruSeq3-SE.fa:2:30:10, HEADCROP:5, TRAILING:20, SLIDINGWINDOW:4:20, MINLEN:15) or paired-end (TruSeq3-PE-2.fa:2:30:10, HEAD CROP:15, TRAILING:20, SLIDINGWINDOW:4:20, MINLEN:15) using the Trimmomatic tool. Surviving reads per sample were then imported into the CLCbio Genomics Workbench and reference mapped by sample against the current instance of the human genome (GRCh38.82) using the "Map Reads To reference" tool supported therein under default parameters (v11). After mapping, alignment files representing pull-downs for different histone markers (27AC, me1, me3) under SBMA condition and WT condition were exported from the Workbench in Binary Alignment Map (BAM) format and then sorted and indexed using samtools. Peak region calling on the sorted alignment files was accomplished using macs2 in conjunction with the "callpeak" command (-B -g hs -q 0.05 --broad --broad-cutoff 0.05). Alignment files representing mock condition and 3 different pull-downs for AR under the SBMA condition and separately under the WT condition were similarly exported from the Workbench, sorted, and then indexed. For peak region calling on these sorted alignments, the macs 2 "callpeak" command was again used but involved in 1 instance, passing to the command all 3 sorted alignment files representing different AR pull-downs under SBMA condition as "treatment" (-t) and the sorted alignment file representing mock as "control" (-c). In a separate instance, all 3 sorted alignment files representing different AR pull-downs under WT condition were passed as "treatment" $(-t)$, and the sorted alignment file representing mock were passed as "control" (-c). For both of these instances, the "callpeak" command parameters used involved the same parameters as those used to call peaks on sorted alignment files representing different histone markers with 1 additional parameter included (-f BAMPE). Fold-enrichment for peak regions called were calculated using the macs 2 "bdgcmp" command, and differential peak regions called between conditions were identified using the macs2 "bdgdiff" command. Distances 
between closest occurring peak regions called between conditions were calculated and summarized using ad hoc commands in R. Characterization of known binding sites occurring within and across peak regions called was accomplished using Genomatix. Heatmaps and density plots were made using EaSeq software package and R studio. IGV Genome Browser was used for peak inspection. GO enrichment analysis of the closest gene with greater than 2-fold reduced H3K27ac was done using Panther (http://pantherdb.org/).

Data availability

The high throughput sequencing data reported in this paper are deposited in NCBI GEO via accession number GSE140018 (https://www.ncbi.nlm.nih.gov/geo/query/acc.cgi?acc=GSE140018).

\section{Statistics}

For all experiments, $n$ denotes technical replicates, whereas $N$ represents biological replicates. For all experiments, 3 SBMA patient, 3 control, and 3 independent differentiation of AR-KO were used. All statistics were performed using Prism. Data are represented as mean \pm SEM. Significance was determined using either 2-tailed Student's $t$ tests or 1-way ANOVA with Bonferroni's test for post hoc analysis. Statistical significance is reported as ${ }^{*} P<0.05,{ }^{* *} P<0.01,{ }^{* *} P<0.001$, and ${ }^{* * * *} P<0.0001$.

\section{Study approval}

Human iPSCs were derived from fibroblasts collected under NIH IRB approved protocol 00-N-0043.

\section{Author contributions}

NP, CG, and KHF designed the experiments. NP, EM, PT, and TE performed the experiments. NP and KJ analyzed the data. NP and EB performed image analysis. ARN provided technical advice on the project. XF worked with the NHLBI iPSC facility to design and characterize the AR-KO iPSCs. NP wrote the first draft of the manuscript, and NP, CG, and KHF edited the manuscript.

\section{Acknowledgments}

We thank Derek Narendra and Vittorio Sartorelli for review and comments on the manuscript. We thank Susan Cheng and Andrea Stanlie for helping with techniques and assay protocols. We thank George Harmison for laboratory support. We thank Jizhong Zou and Kaari Linask (NHLBI iPSC core) for helping to produce the AR-KO iPSCs. This work was supported by intramural research funding from the National Institute of Neurological Disorders and Stroke, NIH.

Address correspondence to: Kenneth H. Fischbeck or Christopher Grunseich, Building 35, Room 2A-1000 (KHF) Room 2A-1010 (CG), 35 Convent Bethesda, Maryland 20892, USA. Phone: 301.435.9318; Email: fischbeck@ninds.nih.gov (KHF). Phone: 301.402.5423; Email: christopher.grunseich@nih.gov (CG).

1. Kennedy WR, Alter M. Progressive proximal spinal and bulbar muscular atrophy of late onset: a sex-linked recessive trait. JClin Neuromuscul Dis. 2000;2(1):3-5.

2. Grunseich C, Rinaldi C, Fischbeck KH. Spinal and bulbar muscular atrophy: pathogenesis and clinical management. Oral Dis. 2014;20(1):6-9.

3. La Spada AR, Wilson EM, Lubahn DB, Harding AE, Fischbeck KH. Androgen receptor gene mutations in X-linked spinal and bulbar muscular atrophy. Nature. 1991;352(6330):77-79.

4. Evans RM. The steroid and thyroid hormone receptor superfamily. Science. 1988;240(4854):889-895.

5. Lieberman AP, Harmison G, Strand AD, Olson JM, Fischbeck KH. Altered transcriptional regulation in cells expressing the expanded polyglutamine androgen receptor. Hum Mol Genet. 2002;11(17):1967-1976

6. McCampbell A, Taye AA, Whitty L, Penney E, Steffan JS, Fischbeck KH. Histone deacetylase inhibitors reduce polyglutamine toxicity. Proc Natl Acad Sci USA. 2001;98(26):15179-15184.

7. Saft C, et al. Mitochondrial impairment in patients and asymptomatic mutation carriers of Huntington's disease. Mov Disord. 2005;20(6):674-679.

8. Malik B, Nirmalananthan N, Gray AL, La Spada AR, Hanna MG, Greensmith L. Co-induction of the heat shock response ameliorates disease progression in a mouse model of human spinal and bulbar muscular atrophy: implications for therapy. Brain. 2013;136(Pt 3):926-943.

9. Theurey P, Rieusset J. Mitochondria-Associated Membranes Response to Nutrient Availability and Role in Metabolic Diseases. Trends Endocrinol Metab. 2017;28(1):32-45

10. Ranganathan S, Harmison GG, Meyertholen K, Pennuto M, Burnett BG, Fischbeck KH. Mitochondrial abnormalities in spinal and bulbar muscular atrophy. Hum Mol Genet. 2009;18(1):27-42. 
11. Palazzolo I, et al. Overexpression of IGF-1 in muscle attenuates disease in a mouse model of spinal and bulbar muscular atrophy. Neuron. 2009;63(3):316-328.

12. Giorgetti E, et al. Rescue of Metabolic Alterations in AR113Q Skeletal Muscle by Peripheral Androgen Receptor Gene Silencing. Cell Rep. 2016;17(1):125-136.

13. Guber RD, et al. Nonalcoholic fatty liver disease in spinal and bulbar muscular atrophy. Neurology. 2017;89(24):2481-2490.

14. Grunseich C, et al. Stem cell-derived motor neurons from spinal and bulbar muscular atrophy patients. Neurobiol Dis. 2014;70:12-20.

15. Fernandopulle MS, Prestil R, Grunseich C, Wang C, Gan L, Ward ME. Transcription Factor-Mediated Differentiation of Human iPSCs into Neurons. Curr Protoc Cell Biol. 2018;79(1):e51.

16. Ho R, et al. ALS disrupts spinal motor neuron maturation and aging pathways within gene co-expression networks. Nat Neurosci. 2016;19(9):1256-1267.

17. Zheng X, et al. Alleviation of neuronal energy deficiency by mTOR inhibition as a treatment for mitochondria-related neurodegeneration. Elife. 2016;5:e13378.

18. Nakano M, Imamura H, Nagai T, Noji H. $\mathrm{Ca}^{2+}$ regulation of mitochondrial ATP synthesis visualized at the single cell level. ACS Chem Biol. 2011;6(7):709-715

19. Stelloo S, et al. Integrative epigenetic taxonomy of primary prostate cancer. Nat Commun. 2018;9(1):4900.

20. Creyghton MP, et al. Histone H3K27ac separates active from poised enhancers and predicts developmental state. Proc Natl Acad Sci USA. 2010;107(50):21931-21936.

21. Bonn S, et al. Tissue-specific analysis of chromatin state identifies temporal signatures of enhancer activity during embryonic development. Nat Genet. 2012;44(2):148-156.

22. Vashishtha M, et al. Targeting H3K4 trimethylation in Huntington disease. Proc Natl Acad Sci USA. 2013;110(32):E3027-E3036

23. Mirabella AC, Foster BM, Bartke T. Chromatin deregulation in disease. Chromosoma. 2016;125(1):75-93.

24. Lee JV, et al. Akt-dependent metabolic reprogramming regulates tumor cell histone acetylation. Cell Metab. 2014;20(2):306-319.

25. Chauvin C, et al. Ribosomal protein S6 kinase activity controls the ribosome biogenesis transcriptional program. Oncogene. 2014;33(4):474-483

26. Shimizu Y, et al. Cell-free translation reconstituted with purified components. Nat Biotechnol. 2001;19(8):751-755

27. Garcia D, Shaw RJ. AMPK: Mechanisms of Cellular Energy Sensing and Restoration of Metabolic Balance. Mol Cell. 2017;66(6):789-800.

28. Vingtdeux V, Davies P, Dickson DW, Marambaud P. AMPK is abnormally activated in tangle- and pre-tangle-bearing neurons in Alzheimer's disease and other tauopathies. Acta Neuropathol. 2011;121(3):337-349.

29. Ju TC, et al. Nuclear translocation of AMPK-alpha1 potentiates striatal neurodegeneration in Huntington's disease. J Cell Biol. 2011;194(2):209-227.

30. Hawley SA, et al. Characterization of the AMP-activated protein kinase kinase from rat liver and identification of threonine 172 as the major site at which it phosphorylates AMP-activated protein kinase. J Biol Chem. 1996;271(44):27879-27887.

31. Dagon Y, Hur E, Zheng B, Wellenstein K, Cantley LC, Kahn BB. p70S6 kinase phosphorylates AMPK on serine 491 to mediate leptin's effect on food intake. Cell Metab. 2012;16(1):104-112.

32. Warden SM, Richardson C, O'Donnell J, Stapleton D, Kemp BE, Witters LA. Post-translational modifications of the beta-1 subunit of AMP-activated protein kinase affect enzyme activity and cellular localization. Biochem J. 2001;354(Pt 2):275-283

33. Chiang GG, Abraham RT. Phosphorylation of mammalian target of rapamycin (mTOR) at Ser-2448 is mediated by p70S6 kinase. J Biol Chem. 2005;280(27):25485-25490.

34. Ashrafi G, Wu Z, Farrell RJ, Ryan TA. GLUT4 Mobilization Supports Energetic Demands of Active Synapses. Neuron. 2017;93(3):606-615.e3.

35. Li Y, et al. AMPK phosphorylates and inhibits SREBP activity to attenuate hepatic steatosis and atherosclerosis in diet-induced insulin-resistant mice. Cell Metab. 2011;13(4):376-388

36. Bengoechea-Alonso MT, Ericsson J. The phosphorylation-dependent regulation of nuclear SREBP1 during mitosis links lipid metabolism and cell growth. Cell Cycle. 2016;15(20):2753-2765.

37. Jin X, Qiao A, Moskophidis D, Mivechi NF. Modulation of Heat Shock Factor 1 Activity through Silencing of Ser303/ Ser307 Phosphorylation Supports a Metabolic Program Leading to Age-Related Obesity and Insulin Resistance. Mol Cell Biol. 2018;38(18):e00095-18.

38. Manning G, Whyte DB, Martinez R, Hunter T, Sudarsanam S. The protein kinase complement of the human genome. Science 2002;298(5600):1912-1934.

39. Nakanishi A, et al. AMP-activated protein kinase-mediated feedback phosphorylation controls the $\mathrm{Ca}^{2+} / \mathrm{calmodulin}(\mathrm{CaM})$ dependence of $\mathrm{Ca}^{2+} / \mathrm{CaM}$-dependent protein kinase kinase $\beta$. J Biol Chem. 2017;292(48):19804-19813.

40. Song C, et al. Elevated Exogenous Pyruvate Potentiates Mesodermal Differentiation through Metabolic Modulation and AMPK/mTOR Pathway in Human Embryonic Stem Cells. Stem Cell Reports. 2019;13(2):338-351.

41. Tie F, Banerjee R, Fu C, Stratton CA, Fang M, Harte PJ. Polycomb inhibits histone acetylation by CBP by binding directly to its catalytic domain. Proc Natl Acad Sci USA. 2016;113(6):E744-E753.

42. Cai L, Sutter BM, Li B, Tu BP. Acetyl-CoA induces cell growth and proliferation by promoting the acetylation of histones at growth genes. Mol Cell. 2011;42(4):426-437.

43. Drazic A, Myklebust LM, Ree R, Arnesen T. The world of protein acetylation. Biochim Biophys Acta. 2016;1864(10):1372-1401.

44. Nagarajan S, Benito E, Fischer A, Johnsen SA. H4K12ac is regulated by estrogen receptor-alpha and is associated with BRD4 function and inducible transcription. Oncotarget. 2015;6(9):7305-7317.

45. Bowers EM, et al. Virtual ligand screening of the p300/CBP histone acetyltransferase: identification of a selective small molecule inhibitor. Chem Biol. 2010;17(5):471-482.

46. Dancy BM, Cole PA. Protein lysine acetylation by p300/CBP. Chem Rev. 2015;115(6):2419-2452.

47. Achour M, et al. Neuronal identity genes regulated by super-enhancers are preferentially down-regulated in the striatum of Huntington's disease mice. Hum Mol Genet. 2015;24(12):3481-3496.

48. Cheng $\mathrm{H}$, et al. Repression of human and mouse brain inflammaging transcriptome by broad gene-body histone hyperacetyla- 
tion. Proc Natl Acad Sci USA. 2018;115(29):7611-7616.

49. Takahashi H, McCaffery JM, Irizarry RA, Boeke JD. Nucleocytosolic acetyl-coenzyme a synthetase is required for histone acetylation and global transcription. Mol Cell. 2006;23(2):207-217.

50. Mews P, Donahue G, Drake AM, Luczak V, Abel T, Berger SL. Acetyl-CoA synthetase regulates histone acetylation and hippocampal memory. Nature. 2017;546(7658):381-386.

51. Shahbazian MD, Grunstein M. Functions of site-specific histone acetylation and deacetylation. Annu Rev Biochem. 2007;76:75-100.

52. Ward JM, et al. Metabolic and Organelle Morphology Defects in Mice and Human Patients Define Spinocerebellar Ataxia Type 7 as a Mitochondrial Disease. Cell Rep. 2019;26(5):1189-1202.e6.

53. Rocchi A, et al. Glycolytic-to-oxidative fiber-type switch and mTOR signaling activation are early-onset features of SBMA muscle modified by high-fat diet. Acta Neuropathol. 2016;132(1):127-144.

54. Han I, You Y, Kordower JH, Brady ST, Morfini GA. Differential vulnerability of neurons in Huntington's disease: the role of cell type-specific features. J Neurochem. 2010;113(5):1073-1091. 